\title{
Grasa y ácidos grasos en leche de vacas pastoreando, en cuatro sistemas de producción ${ }^{1}$
}

\author{
Fat and fatty acids of cow milk grazing in four production systems
}

\author{
Esperanza Prieto-Manrique², Julio Ernesto Vargas-Sánchez ${ }^{3}$,Joaquín Angulo-Arizala ${ }^{4}$, \\ Liliana Mahecha-Ledesma ${ }^{4}$
}

\begin{abstract}
RESUMEN
El objetivo de este trabajo fue evaluar el efecto de la dieta, número de partos y tercio de lactancia sobre el porcentaje de grasa, la concentración de ácido linoleico conjugado (ALC-c9t11), transvaccénico (ATV-C18:1t11) y otros ácidos grasos (AG) insaturados en la leche de vacas pastoreando en cuatro sistemas de producción. El trabajo se realizó de mayo a diciembre del 2012 en diez ganaderías colombianas, representativas de los sistemas de producción lechería tropical (LT) y doble propósito (DP) con/sin sistema silvopastoril intensivo de leucaena (SSPi). En cada ganadería se tomaron tres muestras individuales de leche, de cinco vacas (día $62 \pm 22$, día $147 \pm 22$ y día $227 \pm 22$ de lactancia), conjuntamente se estimó el consumo de suplemento y de forraje, y se determinaron $\mathrm{AG}$ en estos. Cada sistema de producción se analizó independientemente. El contenido de grasa en leche fue afectado por el tercio de lactancia $(p<0,05)$ en LTSSPi y DPSSPi, presentando el primer tercio el menor valor. ALC-c9t11 en la leche varió entre 1,02 a 2,22\%, ATV entre 4,40 a 6,50\% y el índice de aterogenicidad entre 1,69 a 2,89 . La alta participación de la grasa de los suplementos (51 a 84\%) en el total de grasa consumida y su composición, 1levó a variaciones en el perfil de AG de la leche en los sistemas LT, LTSSPi y DP. El número de parto y el tercio de lactancia presentaron menor efecto que la dieta.
\end{abstract}

Palabras clave: ácido linoleico conjugado, ácido transvaccénico, lechería tropical, doble propósito, leucaena.

\begin{abstract}
The aim of this work was to evaluate the effect of diet, number of births and stage of lactation on the percentage of milk fat, concentration of conjugated linoleic acid (CLA-c9t11), trans-vaccenic acid (TVA-C18:1t11) and other milk unsaturated fatty acids (FA) of cows grazing in four production systems. This work was carried out from May to December 2012 in ten farms in Colombia, representing tropical dairy production system (LT) and dual purpose production system (DP) with and without intensive silvopastoral system of leucaena (SSPi). Three individual samples of milk from five cows were taken in each farm (day $62 \pm 22$, day $147 \pm 22$ and day $227 \pm 22$ of lactation), at the same time, supplement and forage intake was estimated and FA were determined in these. Each production system was independently analyzed. The milk fat content was affected by the stage of lactation $(\mathrm{p}<0.05)$ in LTSSPi and DPSSPi, obtaining the lower value in the first stage of lactation. CLA-c9t11 in the milk ranged between 1.02 and $2.22 \%$, TVA between 4.40 and $6.50 \%$ and the atherogenic index between 1.69 and 2.89. The high share of fat supplements (51 to $84 \%$ ) on total fat consumed and the composition of fat supplements affected the variations of the milk fatty acid profile in LT, LTSSPi and DP systems. Births and stage of lactation had a lower effect than dieting.
\end{abstract}

Keywords: conjugated linoleic acid, trans-vaccenic acid, tropical dairy system, dual purpose, leucaena.

1 Recibido: 13 de enero, 2016. Aceptado: 30 de marzo, 2016. Este trabajo formó parte de un proyecto de investigación financiado por COLCIENCIAS-Colombia y es parte de la tesis doctoral del primer autor.

2 Universidad de Sucre, Facultad de Ciencias Agropecuarias. Carrera 28 № 5-267, Barrio Puerta Roja, Sincelejo -Sucre, Colombia. esperanza. prieto@unisucre.edu.co

3 Universidad de Caldas, Facultad de Ciencias Agropecuarias, Departamento Sistemas de Producción. Calle 65 N $^{\circ} 26-10$, Manizales - Caldas, Colombia.jvargas@ucaldas.edu.co

4 Universidad de Antioquia, Facultad de Ciencias Agrarias, Grupo de Investigación en Ciencias Animales (GRICA). Sede Robledo, Carrera 75 N ${ }^{\circ}$ 65-87 bloque 46, Medellín - Antioquia, Colombia. joaquin.angulo@udea.edu.co, liliana.mahecha@udea.edu.co (autor para correspondencia). 


\section{INTRODUCCIÓN}

El ácido linoleico conjugado (ALC-c9t11 o ácido ruménico), el ácido transvaccénico (ATV), algunos ácidos grasos de cadena larga (AGCL) n-3 y la proporción de ácidos grasos (AG) insaturados, de la leche bovina, se relacionan con beneficios para la salud humana (Harris, 2008; Shingfield et al., 2013).

En la leche bovina, ALC-c9t11, ATV, AGCL n-3 y ácidos grasos insaturados resultan del consumo de estos últimos, de la extensión de la biohidrogenación ruminal (BHR) (por bacterias ruminales Butyrivibrio fibrisolvens principalmente (Kepler y Tove, 1967; Wallace et al., 2007)) o de la capacidad para escapar a la misma. La magnitud de las cantidades presentes en la leche está determinada principalmente por factores dietarios (Palmquist, 2007).

El contenido en ALC-c9t11 en la leche es mayor en animales que reciben raciones con una mayor proporción de forraje, puede estar afectado por el tipo de forraje suministrado, y es mayor en animales en pastoreo que cuando reciben forrajes conservados (ensilados) (Weiss et al., 2004 a; b; Mele et al., 2006). El efecto enriquecedor de las pasturas sobre los niveles de ALC-c9t11 en leche, se explica por el consumo de ácido linolénico proveniente del pasto, su posterior conversión en ATV (C18:1 t11) a nivel de rumen y la subsiguiente transformación a CLA-c9t11 por actividad de la enzima mamaria delta-9 desaturasa (Griinari y Bauman, 1999); se estima que más del 74\% de ALC-c9t11 en la grasa de la leche es sintetizado a través de esta vía (Bichi et al., 2012).

Aunque dietas a base de pasturas resultan en leche con mayores niveles basales de ALC-c9t11, se pueden alcanzar aumentos adicionales con la suplementación de lípidos (Chilliard y Ferlay, 2004; Schroeder et al., 2004). La suplementación con ácidos grasos poliinsaturados, permite aumentar ALC-c9t11, ATV, AG insaturados y disminuir la concentración de AG saturados (Ferlay et al., 2013), que presentan efecto aterogénico (Ulbritch y Southgate, 1991) e hipercolesterolémico (Givens, 2010), pero su efecto varía de acuerdo con la cantidad y fuente de grasa empleada y con la composición de la dieta basal (Chillard y Ferlay, 2004; Dewhurst et al., 2006).

En Colombia, el $45 \%$ de la leche se produce en lechería especializada de trópico alto, y el otro 55\% bajo el sistema doble propósito (CONPES, 2010), en donde se incluyen los sistemas de lechería tropical como una modificación del doble propósito pero con mayor tecnología y orientado solo a la producción de leche. Los sistemas doble propósito y lechería tropical, en su expresión tradicional, se manejan bajo pastoreo con una base forrajera de solo gramíneas, sin o con suplementación de alimentos concentrados, con el fin de cubrir los requerimientos nutricionales de las vacas. Sin embargo, otros productores utilizan pastoreo en sistemas con una base forrajera silvopastoril (Murgueitio, 1999; Pagiola et al., 2005; 2007). Hay evidencia de que algunos metabolitos secundarios presentes en plantas forrajeras no gramíneas, pueden afectar la biohidrogenación ruminal (Khiaosa-Ard et al., 2009) y de esta forma podrían modificar la composición de la leche.

El rango en las concentraciones de ALC-c9t11 en la leche de las vacas bajo pastoreo es amplio (0,5 a 1,7\%), debido a cambios estacionales en la disponibilidad de pasto, asignación de pastura, composición botánica de los recursos naturales y pastos cultivados, concentración de lípidos y ácido linolénico (Chilliard y Ferlay, 2004; Schroeder et al., 2004), y al tipo de suplemento ofrecido (Rico et al., 2007); aunque los factores dietarios son los principales determinantes de las concentraciones de ALC-c9t11 en leche, otros factores como el parto y el estado de lactación también presentan influencia (Lawless et al., 1999; White et al., 2001; Kelsey et al., 2003). En Colombia existe muy poca información al respecto; por lo tanto, el desarrollo de estrategias de suplementación en la ganadería colombiana, con ácidos grasos poliinsaturados que permitan aumentar los ácidos grasos benéficos en la leche, exige un previo conocimiento de las condiciones de producción y alimentación específicas del país; que varían de acuerdo con el sistema de producción.

El objetivo de este trabajo fue evaluar el efecto de la dieta, número de partos y tercio de la lactancia sobre la grasa y el perfil de ácidos grasos en la leche de vacas pastoreando en cuatro sistemas de producción.

\section{MATERIALES Y MÉTODOS}

\section{Localización}

Este trabajo se llevó a cabo en diez ganaderías (fincas) de Colombia. Se seleccionaron cuatro fincas representativas del sistema de producción doble propósito (DP), definido en su forma más universal 
de ordeño con becerro y cruces mixtos de Bos taurus $\mathrm{x}$ Bos indicus, bajo pastoreo sin sistema silvopastoril (dos fincas) y con sistemas silvopastoril intensivos (SSPi) (dos fincas); además, del sistema de producción lechería tropical (LT), se seleccionaron seis definidas por el predominio de animales Bos taurus, el ordeño sin ternero, bajo pastoreo sin SSPi (tres fincas) y con SSPi (tres fincas). La localización y características generales de las fincas se presentan en el Cuadro 1.

El sistema silvopastoril intensivo se definió como el uso de la tierra destinado a la producción tropical eficiente y natural de ganado bovino, donde se combina en el mismo espacio una mezcla de gramíneas mejoradas (estoloníferas y cespitosas), con un segundo estrato de plantas de Leucaena leucocephala u otras, no menor a 8000 arbustos por hectárea (Murgueitio e Ibrahim, 2008).
Los sistemas de alimentación fueron: bajo pastoreo de solo gramíneas (Cynodon plectostachyus y/o Panicum máximum (Megathyrsus maximus) cv. Tanzania) y bajo pastoreo en sistemas silvopastoriles (Cynodon plectostachyus y/o Panicum máximum (Megathyrsus maximus) cv. Tanzania con Leucaena leucocephala).

Se evaluó el efecto de la dieta propia de cada finca, el número de partos y el tercio de lactancia sobre el porcentaje de grasa, la concentración de ALC-c9t11, ATV y otros AGCL en la leche.

Los productores de las fincas seleccionadas realizan pastoreo rotacional con periodo de ocupación entre 12 y 48 horas, con disponibilidad de agua fresca y limpia para los animales; en las fincas de los sistemas LT, LTSSPi y DP se suplementa la dieta base de acuerdo con la producción de leche/animal/día, con alimento

Cuadro 1. Localización y características generales de las fincas donde se evaluó la grasa y los ácidos grasos en la leche de vacas bajo pastoreo en sistemas con/sin silvopasturas intensivas de leucaena, en el periodo mayo-diciembre 2012. Colombia.

Table 1. Location and general characteristics of the farms where fat and fatty acids were evaluated in the milk of cows grazing on systems with / without intensive silvopastures of leucaena, during May-December 2012. Colombia.

\begin{tabular}{|c|c|c|c|c|c|c|c|c|c|c|}
\hline $\mathbf{N}^{\circ}$ & Sistema & Finca & $\begin{array}{c}\text { Municipio y } \\
\text { departamento }\end{array}$ & $\begin{array}{l}\text { Altitud } \\
\text { (m) }\end{array}$ & $\begin{array}{l}\text { T.P. }{ }^{1} \\
\left({ }^{\circ} \mathrm{C}\right)\end{array}$ & Forraje & $\begin{array}{c}\text { D.D }^{2} \\
\text { pastura }\end{array}$ & $\begin{array}{l}\text { Leche } \\
\text { l/día }{ }^{3}\end{array}$ & $\begin{array}{c}\text { Tipo } \\
\text { ordeño }\end{array}$ & $\begin{array}{c}\text { Peso } \\
\text { vacas }(k g)\end{array}$ \\
\hline 1 & LT & Esperanza & Risaralda - Caldas & 1743 & 21 & Estrella & 30 & 15,4 & Mecánico & 500 \\
\hline 2 & LT & Pradera & Risaralda - Caldas & 1743 & 21 & Guinea & 32 & 12,2 & Mecánico & 500 \\
\hline 3 & LT & San Felipe & Pereira-Risaralda & 1411 & 22 & Estrella & 27 & 11,8 & Mecánico & 450 \\
\hline 4 & LTSSPi & Asturias & Tebaida - Quindío & 1190 & 23 & $\begin{array}{c}\text { Estrella } \\
\text { Guinea } \\
\text { Leucaena }\end{array}$ & 43 & 15,6 & Mecánico & 500 \\
\hline 5 & LTSSPi & Hatico & $\begin{array}{c}\text { Cerrito-Valle del } \\
\text { Cauca }\end{array}$ & 987 & 24 & $\begin{array}{c}\text { Estrella } \\
\text { Guinea } \\
\text { Leucaena }\end{array}$ & 51 & 13,4 & Mecánico & 380 \\
\hline 6 & LTSSPi & Lucerna & $\begin{array}{l}\text { Buga la Grande- } \\
\text { Valle del Cauca }\end{array}$ & 941 & 23 & $\begin{array}{c}\text { Estrella } \\
\text { Leucaena }\end{array}$ & 41 & 15,6 & Mecánico & 420 \\
\hline 7 & $\mathrm{DP}^{4}$ & Maracaibo & Neira - Caldas & 1969 & 18 & Estrella & 27 & 10,8 & Mecánico & 550 \\
\hline 8 & $\mathrm{DP}^{4}$ & Vargas & Palestina - Caldas & 1630 & 23 & $\begin{array}{l}\text { Estrella } \\
\text { Guinea }\end{array}$ & 26 & 9,3 & Mecánico & 500 \\
\hline 9 & DPSSPi $^{5}$ & Pradera & $\begin{array}{c}\text { La Jagua del Pilar } \\
\text { - Guajira }\end{array}$ & 223 & 28 & $\begin{array}{c}\text { Guinea } \\
\text { Leucaena }\end{array}$ & 31 & 6,2 & Manual & 440 \\
\hline 10 & $\operatorname{DPSSPi}^{5}$ & Salsipuedes & Fonseca - Guajira & 12 & 28 & $\begin{array}{c}\text { Guinea } \\
\text { Leucaena }\end{array}$ & 47 & 5,5 & Manual & 400 \\
\hline
\end{tabular}

LT: lechería tropical; DP: Doble propósito; SSPi: sistema silvopastoril intensivo / LT: tropical dairy; DP: dual purpose; SSPi: intensive silvopastoral system.

${ }^{1}$ T.P: temperatura promedio / T.P: average temperature.

${ }^{2}$ D.D: días de descanso / D.D: rest days.

${ }^{3}$ Promedio de los tres muestreos, del grupo de vacas en estudio / Average of three samples from the study group of cows.

${ }^{4}$ Terneros menores de tres meses permanecen con la vaca tres horas/día y los mayores de tres meses solo reciben la leche de la madre en el ordeño / Calves under three months stay with the cow three hours / day and those over three months only receive their mother's milk at milking. ${ }^{5}$ Terneros menores de cuatro meses permanecen con la vaca ocho horas/día y los mayores de cuatro meses solo reciben la leche de la madre en el ordeño / Calves under four months stay with the cow eight hours / day and those over four months only receive their mother's milk at milking. 
concentrado y/o subproductos de la agroindustria o una mezcla de los dos, en el sistema DPSSPi no se suplementa y en todos los sistemas ofrecen sal mineralizada.

En cada finca, se seleccionaron cinco vacas que estaban entre uno y siete partos, razón por la cual se evaluó el efecto del número de partos. El peso promedio de estas vacas y el tipo de ordeño, se presenta en el Cuadro 1. En las vacas se tomaron muestras individuales de leche, durante tres veces (día $62 \pm 22$, día $147 \pm 22$ y día $227 \pm 22$ de lactancia), en el periodo de lluvias (mayo - inicio de diciembre). Las muestras se tomaron por triplicado: una para análisis de grasa total, una para perfil de ácidos grasos y una contramuestra.

Conjuntamente con cada uno de los muestreos de leche, se estimó el consumo de materia seca del suplemento, mediante oferta y rechazo, y del forraje, mediante la técnica de desaparición de forraje
(Macoon et al., 2003). Para la leucaena, se adaptó esta metodología y se estimó la disponibilidad de forraje por metro lineal (Mahecha et al., 2000). Seguidamente, se tomaron muestras de cada componente de la dieta para determinar la composición química, porcentaje de grasa y el perfil de ácidos grasos (Cuadros 2, 3 y 4). Con base en lo anterior, se estimó el consumo diario por animal de grasa y de ácidos grasos oleico, linoleico y linolénico (Cuadros 5 y 6 ).

El análisis de grasa total de la leche se realizó en la leche fresca. Las muestras de leche y alimentos para perfil de ácidos grasos, se mantuvieron congeladas a $-20{ }^{\circ} \mathrm{C}$ hasta la realización del análisis (Christie, 1990).

Los AG de la leche se analizaron mediante cromatografía de gases con detector de ionización de llama (FID), siguiendo la metodología propuesta por Tequin-Ocampo (2014), tanto para la extracción-

Cuadro 2. Composición química y ácidos grasos de los forrajes ofrecidos a vacas bajo pastoreo en los sistemas lechería tropical con/ sin sistema silvopastoril intensivo de leucaena, en el periodo mayo-diciembre 2012. Colombia.

Table 2. Chemical composition and fatty acids of forage offered to cows grazing in tropical dairy production systems with / without intensive silvopastoral system of leucaena, during May-December 2012. Colombia.

\begin{tabular}{|c|c|c|c|c|c|c|c|c|c|}
\hline \multirow[t]{3}{*}{ Variable } & \multicolumn{3}{|c|}{ Lechería tropical } & \multicolumn{6}{|c|}{ Lechería tropical SSPi } \\
\hline & Esperanza & Pradera & San Felipe & Asturias & Hatico & Lucerna & Asturias & Hatico & Lucerna \\
\hline & Estrella & Guinea & Estrella & Estrella & Estrella & Estrella & Leucaena & Leucaena & Leucaena \\
\hline Grasa $\%$ & 1,5 & 0,8 & 1,2 & 1,1 & 0,9 & 1,0 & 2,0 & 2,6 & 2,3 \\
\hline Proteína \% & 16,2 & 11,5 & 12,2 & 11,9 & 8,9 & 8,7 & 26,0 & 27,6 & 27,3 \\
\hline FDN \% & 64,3 & 65,7 & 69,2 & 69,0 & 69,1 & 72,9 & 31,4 & 31,5 & 28,8 \\
\hline FDA \% & 28,7 & 37,2 & 31,5 & 32,0 & 34,0 & 35,1 & 14,4 & 17,2 & 16,0 \\
\hline \multirow[t]{2}{*}{ Cenizas $\%$} & 9,6 & 14,4 & 8,4 & 10,2 & 9,7 & 9,4 & 6,9 & 8,3 & 7,7 \\
\hline & \multicolumn{9}{|c|}{ g AG/100g AG totales } \\
\hline $\mathrm{C} 14: 0$ & 0,44 & 0,61 & 0,60 & 0,59 & 0,76 & 0,59 & 0,30 & 0,55 & 0,52 \\
\hline C16:0 & 24,78 & 29,51 & 26,00 & 27,20 & 27,85 & 25,91 & 24,46 & 24,10 & 24,97 \\
\hline C18:0 & 1,75 & 2,45 & 2,05 & 2,15 & 2,53 & 2,08 & 5,20 & 6,21 & 7,20 \\
\hline C18:1c9 & 1,79 & 2,05 & 2,06 & 2,07 & 2,38 & 1,94 & 2,15 & 3,04 & 3,36 \\
\hline $\mathrm{C} 18: 2 \mathrm{c} 9,12$ & 15,20 & 17,33 & 15,94 & 16,03 & 16,87 & 15,80 & 16,29 & 16,04 & 14,01 \\
\hline $\mathrm{C} 18: 3 \mathrm{c} 9,12,15$ & 47,26 & 39,04 & 41,61 & 41,70 & 35,84 & 41,70 & 42,54 & 39,62 & 38,08 \\
\hline \multicolumn{10}{|l|}{ Sumatorias } \\
\hline Saturados & 30,95 & 36,98 & 34,52 & 34,89 & 38,48 & 34,62 & 33,14 & 35,39 & 37,19 \\
\hline Insaturados & 69,04 & 63,01 & 65,47 & 65,10 & 61,51 & 65,37 & 66,85 & 64,60 & 62,80 \\
\hline Monoinsaturados & 4,76 & 4,96 & 5,75 & 5,38 & 6,13 & 5,84 & 6,89 & 7,63 & 9,44 \\
\hline Poliinsaturados & 64,28 & 58,05 & 59,72 & 59,71 & 55,13 & 59,53 & 59,95 & 56,97 & 53,36 \\
\hline
\end{tabular}

SSPi: sistema silvopastoril intensivo / SSPi= intensive silvopastoral system. 
Cuadro 3. Composición química y ácidos grasos de los forrajes ofrecidos a vacas bajo pastoreo en los sistemas doble propósito con/sin sistema silvopastoril intensivo de leucaena, en el periodo mayo-diciembre 2012. Colombia.

Table 3. Chemical composition and fatty acids of forage offered to cows grazing in dual purpose production systems with/without intensive silvopastoral systems of leucaena, during May-December 2012. Colombia.

\begin{tabular}{|c|c|c|c|c|c|c|}
\hline \multirow[t]{3}{*}{ Variable } & \multicolumn{2}{|c|}{ Doble propósito } & \multicolumn{4}{|c|}{ Doble propósito SSPi } \\
\hline & Maracaibo & Vargas & Pradera & Salsipuedes & Pradera & Salsipuedes \\
\hline & Estrella & Estrella & Guinea & Guinea & Leucaena & Leucaena \\
\hline Grasa \% & 1,2 & 1,0 & 1,2 & 1,2 & 2,7 & 3,5 \\
\hline Proteína \% & 13,8 & 15,7 & 9,6 & 7,8 & 28,6 & 24,5 \\
\hline FDN $\%$ & 69,7 & 68,2 & 71,1 & 72,0 & 33,0 & 29,9 \\
\hline FDA $\%$ & 32,6 & 31,7 & 37,8 & 38,6 & 17,0 & 15,3 \\
\hline \multirow{2}{*}{ Cenizas \% } & 10,6 & 11,1 & 12,2 & 11,5 & 8,2 & 7,4 \\
\hline & \multicolumn{6}{|c|}{ g AG/100g AG totales } \\
\hline C14:0 & 0,58 & 0,53 & 0,86 & 0,67 & 0,33 & 0,35 \\
\hline C16:0 & 25,32 & 26,30 & 27,42 & 27,34 & 22,77 & 21,63 \\
\hline C18:0 & 1,72 & 1,93 & 3,13 & 2,45 & 6,43 & 6,33 \\
\hline C18:1c9 & 2,17 & 1,95 & 3,61 & 1,83 & 2,99 & 3,15 \\
\hline $\mathrm{C} 18: 2 \mathrm{c} 9,12$ & 15,99 & 15,99 & 14,31 & 15,39 & 14,17 & 11,41 \\
\hline $\mathrm{C} 18: 3 \mathrm{c} 9,12,15$ & 43,95 & 44,09 & 43,69 & 43,21 & 43,20 & 47,39 \\
\hline \multicolumn{7}{|l|}{ Sumatorias } \\
\hline Saturados & 32,63 & 32,97 & 34,49 & 34,65 & 32,80 & 31,38 \\
\hline Insaturados & 67,36 & 67,02 & 65,50 & 65,34 & 67,19 & 68,61 \\
\hline Monoinsaturados & 5,51 & 5,17 & 6,12 & 4,87 & 8,52 & 8,63 \\
\hline Poliinsaturados & 61,85 & 61,85 & 59,38 & 60,47 & 58,67 & 59,98 \\
\hline
\end{tabular}

SSPi: sistema silvopastoril intensivo / SSPi= intensive silvopastoral system.

Cuadro 4. Composición química y ácidos grasos de los suplementos ofrecidos a vacas bajo pastoreo en los diferentes sistemas de producción, en el periodo mayo-diciembre 2012. Colombia.

Table 4. Fatty acids and chemical composition of supplements offered to cows grazing in different production systems, during May-December 2012. Colombia.

\begin{tabular}{|c|c|c|c|c|c|c|c|c|}
\hline \multirow[t]{2}{*}{ Variable } & \multicolumn{3}{|c|}{ Lechería tropical } & \multicolumn{3}{|c|}{ Lechería tropical SSPi } & \multicolumn{2}{|c|}{ Doble propósito } \\
\hline & Esperanza & Pradera & San Felipe & Asturias & Hatico & Lucerna & Maracaibo & Vargas \\
\hline Grasa $\%$ & 5,7 & 4,2 & 4,0 & 7,3 & 10,0 & 5,9 & 7,9 & 3,8 \\
\hline Proteína \% & 17,1 & 18,0 & 20,5 & 15,8 & 12,2 & 12,8 & 19,7 & 17,3 \\
\hline FDN \% & 32,0 & 36,7 & 26,0 & 32,7 & 19,6 & 24,1 & 36,5 & 36,3 \\
\hline FDA $\%$ & 10,0 & 16,2 & 7,3 & 11,4 & 5,8 & 6,5 & 18,5 & 14,5 \\
\hline \multirow[t]{2}{*}{ Cenizas \% } & 6,4 & 9,0 & 8,2 & 9,5 & 7,0 & 5,8 & 3,7 & 7,5 \\
\hline & \multicolumn{8}{|c|}{ g AG/100g AG totales } \\
\hline $\mathrm{C} 16: 0$ & 22,62 & 26,70 & 19,70 & 27,88 & 25,88 & 24,24 & 28,33 & 21,47 \\
\hline C18:0 & 2,56 & 2,69 & 3,06 & 8,76 & 5,33 & 11,01 & 3,00 & 3,43 \\
\hline C18:1c9 & 23,73 & 21,43 & 23,94 & 22,60 & 33,56 & 26,45 & 19,00 & 21,80 \\
\hline $\mathrm{C} 18: 2 \mathrm{c} 9,12$ & 44,83 & 40,41 & 44,35 & 28,65 & 27,67 & 25,93 & 44,90 & 36,20 \\
\hline $\mathrm{C} 18: 3 \mathrm{c} 9,12,15$ & 2,24 & 1,59 & 4,26 & 1,60 & 1,50 & 1,33 & 0,99 & 1,97 \\
\hline \multicolumn{9}{|l|}{ Sumatorias } \\
\hline Saturados & 27,60 & 35,43 & 25,26 & 43,13 & 34,77 & 41,14 & 33,63 & 37,90 \\
\hline Insaturados & 72,39 & 64,56 & 74,73 & 56,86 & 65,22 & 58,85 & 66,36 & 62,09 \\
\hline Monoinsaturados & 24,88 & 22,27 & 25,62 & 25,89 & 35,56 & 30,57 & 20,23 & 23,47 \\
\hline Poliinsaturados & 47,51 & 42,28 & 49,11 & 30,97 & 29,65 & 28,27 & 46,13 & 38,62 \\
\hline
\end{tabular}

SSPi: sistema silvopastoril intensivo / SSPi: intensive silvopastoral system. 
Cuadro 5. Consumo estimado de forraje y de ácidos grasos oleico, linoleico y linolénico en los sistemas de lechería tropical con/sin sistema silvopastoril intensivo de leucaena, en el periodo mayo-diciembre 2012. Colombia.

Table 5. Estimated consumption of forages and oleic, linoleic and linolenic fatty acids in the tropical dairy production systems with / without intensive silvopastoral system of leucaena, during May-December 2012. Colombia.

\begin{tabular}{|c|c|c|c|c|c|c|}
\hline \multirow[t]{2}{*}{ Variable } & \multicolumn{3}{|c|}{ LT } & \multicolumn{3}{|c|}{ LTSSPi } \\
\hline & Esperanza & Pradera & San Felipe & Asturias & Hatico & Lucerna \\
\hline & \multicolumn{6}{|c|}{ Consumo kg MS/a/d } \\
\hline Pasto & 8,60 & 8,20 & 8,09 & 9,27 & 4,82 & 6,09 \\
\hline Leucaena & 0,00 & 0,00 & 0,00 & 2,62 & 0,78 & 2,14 \\
\hline Concentrado & 3,42 & 5,22 & 3,69 & 5,04 & 3,33 & 4,59 \\
\hline Total & 12,02 & 13,42 & 11,78 & 16,93 & 8,93 & 12,83 \\
\hline$\%$ forraje & 72 & 61 & 69 & 70 & 63 & 64 \\
\hline$\%$ concentrado & 28 & 39 & 31 & 30 & 37 & 36 \\
\hline \% gramínea & 100 & 100 & 100 & 78 & 86 & 74 \\
\hline \multirow[t]{2}{*}{$\%$ Leucaena } & 0 & 0 & 0 & 22 & 14 & 26 \\
\hline & \multicolumn{6}{|c|}{ Consumo grasa kg/a/d } \\
\hline Pasto & 0,129 & 0,069 & 0,094 & 0,103 & 0,043 & 0,058 \\
\hline Leucaena & & & & 0,052 & 0,021 & 0,050 \\
\hline Concentrado & 0,196 & 0,220 & 0,148 & 0,370 & 0,334 & 0,269 \\
\hline Total & 0,325 & 0,288 & 0,242 & 0,525 & 0,397 & 0,377 \\
\hline$\%$ de la MS & 2,70 & 2,15 & 2,05 & 3,10 & 4,44 & 2,94 \\
\hline \multicolumn{7}{|l|}{ Aporte grasa \% } \\
\hline Forraje & 39,6 & 23,8 & 38,8 & 29,5 & 16,0 & 28,6 \\
\hline \multirow{2}{*}{ Concentrado } & 60,4 & 76,2 & 61,2 & 70,5 & 84,0 & 71,4 \\
\hline & \multicolumn{6}{|c|}{ Consumo del AG (\%) del total de grasa consumida } \\
\hline \multicolumn{7}{|c|}{ ( } \\
\hline Pasto & 0,71 & 0,49 & 0,80 & 0,41 & 0,26 & 0,30 \\
\hline Leucaena & & & & 0,21 & 0,16 & 0,44 \\
\hline Concentrado & 14,32 & 16,32 & 14,65 & 15,93 & 28,20 & 18,89 \\
\hline \multicolumn{7}{|l|}{ Linoleico } \\
\hline Pasto & 6,03 & 4,13 & 6,19 & 3,14 & 1,82 & 2,43 \\
\hline Leucaena & & & & 1,61 & 0,83 & 1,85 \\
\hline Concentrado & 27,06 & 30,78 & 27,14 & 20,20 & 23,25 & 18,52 \\
\hline \multicolumn{7}{|l|}{ Linolénico } \\
\hline Pasto & 18,74 & 9,31 & 16,15 & 8,18 & 3,87 & 6,42 \\
\hline Leucaena & & & & 4,21 & 2,05 & 5,03 \\
\hline Concentrado & 1,35 & 1,21 & 2,61 & 1,13 & 1,26 & 0,95 \\
\hline \multirow[t]{2}{*}{ Total } & 68,20 & 62,24 & 67,53 & 55,02 & 61,70 & 54,82 \\
\hline & \multicolumn{6}{|c|}{ Participación de cada AG \% } \\
\hline Oleico & 15,03 & 16,81 & 15,45 & 16,55 & 28,61 & 19,63 \\
\hline linoleico & 33,08 & 34,91 & 33,32 & 24,95 & 25,90 & 22,80 \\
\hline linolénico & 20,09 & 10,52 & 18,75 & 13,51 & 7,18 & 12,40 \\
\hline
\end{tabular}

LT: lechería tropical, SSPi: sistema silvopastoril intensivo / LT: tropical dairy, SSPi: intensive silvopastoral system.

derivatización como para el análisis cromatográfico en sí mismo. Para esto, la muestra se descongeló, se homogenizó vigorosamente con la mano y luego, por tres minutos en vortex, se transfirieron $300 \mathrm{ul}$ a viales de $4 \mathrm{ml}$ ámbar con tapa de sílica y se liofilizaron. La derivatización de los ácidos grasos y extracción de los metil éster de ácidos grasos, se llevó a cabo a partir de esta muestra, siguiendo el protocolo de trans-esterificación mediante el siguiente procedimiento: tomar $300 \mathrm{ul}$ de leche liofilizada en viales de reacción ámbar de $4 \mathrm{ml}$ con tapas de sílica; adicionar 300 ppm de estándar interno C19; adicionar $1 \mathrm{ml}$ de metóxido de 
Cuadro 6. Consumo estimado de forraje y de ácidos grasos oleico, linoleico y linolénico en los sistemas doble propósito con/sin sistema silvopastoril intensivo de leucaena, en el periodo mayo-diciembre 2012. Colombia.

Table 6. Estimated consumption of forages and oleic, linoleic and linolenic fatty acids in the dual purpose production systems with / without intensive silvopastoral system of leucaena, during May-December 2012. Colombia.

\begin{tabular}{|c|c|c|c|c|}
\hline \multirow[t]{2}{*}{ Variable } & \multicolumn{2}{|c|}{ DP } & \multicolumn{2}{|c|}{ DPSSPi } \\
\hline & Maracaibo & Vargas & Pradera & Salsipuedes \\
\hline & \multicolumn{4}{|c|}{ Consumo kg MS/a/d } \\
\hline Pasto & 9,92 & 8,80 & 8,72 & 7,99 \\
\hline Leucaena & 0,00 & 0,00 & 1,66 & 0,25 \\
\hline Concentrado & 2,52 & 2,43 & 0,0 & 0,0 \\
\hline Total & 12,44 & 11,23 & 10,38 & 8,23 \\
\hline$\%$ forraje & 80 & 78 & 100 & 100 \\
\hline$\%$ concentrado & 20 & 22 & 0 & 0 \\
\hline \% gramínea & 100 & 100 & 84 & 97 \\
\hline \multirow[t]{2}{*}{$\%$ leucaena } & 0 & 0 & 16 & 3 \\
\hline & \multicolumn{4}{|c|}{ Consumo grasa kg/a/d } \\
\hline Pasto & 0,119 & 0,090 & 0,109 & 0,098 \\
\hline Leucaena & & & 0,045 & 0,009 \\
\hline Concentrado & 0,200 & 0,093 & 0,000 & 0,000 \\
\hline Total & 0,319 & 0,183 & 0,154 & 0,107 \\
\hline$\%$ de la MS & 2,56 & 1,63 & 1,48 & 1,30 \\
\hline \multicolumn{5}{|l|}{ Aporte grasa $\%$} \\
\hline Forraje & 37,3 & 49,1 & 100 & 100 \\
\hline \multirow[t]{2}{*}{ Concentrado } & 62,7 & 50,9 & 0,0 & 0,0 \\
\hline & \multicolumn{4}{|c|}{$\begin{array}{c}\text { Consumo del AG }(\%) \text { del total de grasa } \\
\text { consumida }\end{array}$} \\
\hline \multicolumn{5}{|l|}{ Oleico } \\
\hline Pasto & 0,81 & 0,96 & 2,55 & 1,68 \\
\hline Leucaena & & & 0,88 & 0,26 \\
\hline Concentrado & 11,91 & 11,10 & & \\
\hline \multicolumn{5}{|l|}{ Linoleico } \\
\hline Pasto & 5,97 & 7,85 & 10,12 & 14,13 \\
\hline Leucaena & & & 4,15 & 0,94 \\
\hline Concentrado & 28,15 & 18,43 & & \\
\hline \multicolumn{5}{|l|}{ Linolénico } \\
\hline Pasto & 16,40 & 21,65 & 30,90 & 39,67 \\
\hline Leucaena & & & 12,65 & 3,89 \\
\hline Concentrado & 0,62 & 1,00 & & \\
\hline \multirow[t]{2}{*}{ Total } & 63,85 & 60,98 & 61,24 & 60,55 \\
\hline & \multicolumn{4}{|c|}{ Participación de cada AG \% } \\
\hline Oleico & 12,72 & 12,05 & 3,43 & 1,94 \\
\hline linoleico & 34,11 & 26,28 & 14,27 & 15,06 \\
\hline linolénico & 17,02 & 22,65 & 43,55 & 43,55 \\
\hline
\end{tabular}

DP: doble propósito; SSPi: sistema silvopastoril intensivo / DP: dual purpose; SSPi: intensive silvopastoral system. sodio $0,5 \mathrm{~N}$ en metanol y mezclar en vortex durante tres minutos, calentar durante diez minutos a $50{ }^{\circ} \mathrm{C}$, sacar y mezclar; calentar a $50{ }^{\circ} \mathrm{C}$ por $20 \mathrm{~min}$, sacar y mezclar; enfriar a temperatura ambiente; adicionar $1 \mathrm{ml}$ de hexano y tapar; mezclar en vortex por 2 min y transferir con pipeta Pasteur la capa superior a viales de $1,5 \mathrm{ml}$ para posterior análisis en cromatografía.

Se utilizó un cromatógrafo de gases con automuestreador. Las condiciones cromatográficas fueron las siguientes: a) fase móvil: gas transportador de nitrógeno, flujo de columna $1 \mathrm{ml} / \mathrm{min}$, velocidad lineal $26 \mathrm{~cm} / \mathrm{seg}$; b) inyector: temperatura $220{ }^{\circ} \mathrm{C}$, volumen 0,2 ul, modo splitt, radio splitt $1: 50 ; \mathrm{c}$ ) columna: modelo CP-Sil-88, longitud $100 \mathrm{~m}$, diámetro interno $0,25 \mathrm{~mm}$, espesor de la película $0,2 \mathrm{ul}$; d) rampa de temperatura: temperatura $150{ }^{\circ} \mathrm{C}$, tiempo de calentamiento tres minutos, rata $15{ }^{\circ} \mathrm{C} / \mathrm{min}$; e) detector: FID, temperatura $250{ }^{\circ} \mathrm{C}$, flujo de $\mathrm{H}_{2} 40 \mathrm{ml} /$ min, flujo de aire $400 \mathrm{ml} / \mathrm{min}$, flujo de $\mathrm{N} 10 \mathrm{ml} / \mathrm{min}$. Los ácidos grasos fueron separados e identificados por comparación de los tiempos de retención con sus respectivos estándares, se cuantificaron utilizando la curva de calibración de los estándares de ALC y sus isómeros (ALC-c9t11; ALC-c10t12; ALC-t10c12 y ALC mezcla para los isómeros restantes), de ATV y los demás ácidos grasos; además, se utilizó como estándar interno ácido nonadecanoico (C19:0). El porcentaje de cada AG fue calculado a partir de su concentración (ppm), determinada por cromatografía (Tequin-Ocampo, 2014).

Con base en el porcentaje de cada ácido graso se calculó, mediante sumatorias, el porcentaje de AG saturados, insaturados, monoinsaturados, poliinsaturados, omega $3(\mathrm{C} 18: 3 \mathrm{c} 9,12,15 ; \mathrm{C} 20: 3 \mathrm{c} 11$, 14, 17; C20:5c5, 8, 11, 14, 17), omega 6 (C18:2t9, 12; C18:2c9, 12; C18:3c6, 9, 12; C18:2c10, t12; C18:2 t10, c12; C20:3c8, 11, 14; C20:4c5, 8, 11, 14; C22:2 c13, 16), relación n6/n3, C10 a C16, trans $\mathrm{C} 18: 1$ (ATV y C18:1t9), aterogénicos (C12:0,C14:0,C16:0) e índice de aterogenicidad. El índice aterogénico (IA) fue calculado de acuerdo con Ulbricht y Southgate (1991), mediante la ecuación IA = C12:0+ 4C14:0 + C16:0/AG insaturados.

Para la determinación de la composición química de los alimentos, se utilizaron las técnicas analíticas convencionales de la AOAC (1999) (materia seca 
método ID 934.01, cenizas método ID 92.05, proteína bruta método ID 984.13 , grasa y fibra detergente ácido método ID 973.18) y los descritos por Van-Soest et al. (1991) para los análisis de fibra detergente neutro y fibra detergente ácido.

El perfil de ácidos grasos de los alimentos, se realizó mediante el método de cromatografía de gases acoplado a espectrometría de masas, siguiendo la metodología propuesta por Tequin-Ocampo (2014), tanto para la extracción-derivatización como para el análisis cromatográfico en sí mismo. Para esto, $15 \mathrm{~g}$ de muestra de alimento fue liofilizada; seguidamente, se realizó la derivatización de los ácidos grasos siguiendo el protocolo de esterificación que consiste en: pesar $50 \mathrm{mg}$ alimento y depositarlos en viales de reacción ámbar de $4 \mathrm{ml}$ con tapas de silica; adicionar 300 ppm de estándar interno C19; adicionar $1 \mathrm{ml}$ de metóxido de sodio $0,5 \mathrm{~N}$ en metanol y mezclar en vortex durante tres minutos; calentar durante diez minutos a $50{ }^{\circ} \mathrm{C}$, sacar y mezclar; adicionar $1 \mathrm{ml}$ de $\mathrm{HCl} 0,5 \mathrm{~N}$ en metanol y mezclar en vortex durante tres minutos; calentar durante diez minutos a $50{ }^{\circ} \mathrm{C}$, sacar y mezclar; enfriar a temperatura ambiente, adicionar $1 \mathrm{ml}$ de hexano y tapar, mezclar en vortex por dos minutos; finalmente, transferir con pipeta Pasteur la capa superior a viales de $1,5 \mathrm{ml}$ para posterior análisis cromatográfico. Se utilizó un cromatógrafo de gases acoplado a espectrómetro de masas, con automuestreador.

Las condiciones cromatográficas fueron las siguientes: a) fase móvil: gas transportador helio, flujo de columna $1 \mathrm{ml} / \mathrm{min}$, velocidad lineal $26 \mathrm{~cm} / \mathrm{seg}$; b) inyector: temperatura $220{ }^{\circ} \mathrm{C}$, volumen $0,2 \mathrm{ul}$, modo splitles; c) columna: modelo CP-Sil-88, longitud 100 $\mathrm{m}$, diámetro interno $0,25 \mathrm{~mm}$, espesor de la película $0,2 \mathrm{ul}$; d) rampa de temperatura: temperatura $150{ }^{\circ} \mathrm{C}$, tiempo de calentamiento tres minutos, rata $15^{\circ} \mathrm{C} / \mathrm{min}$; e) detector: temperatura $250^{\circ} \mathrm{C}$, flujo de $\mathrm{N}_{2} 10 \mathrm{ml} / \mathrm{min}$. Los ácidos grasos fueron separados e identificados siguiendo el mismo procedimiento que se utilizó para ácidos grasos de la leche, descrito anteriormente.

\section{Análisis estadístico}

El análisis para las variables contenido de grasa, perfil de ácidos grasos de la leche y las respectivas sumatorias, se realizó mediante un modelo mixto de medidas repetidas para cada sistema de forma independiente, que incluyó el efecto de la dieta propia de cada finca (finca), tercio de lactancia, vaca dentro de finca, número de partos y sus interacciones, utilizando PROC MIXED de SAS (SAS, 2001). La diferencia entre promedios, se analizó mediante prueba de Tuckey-Kramer, con nivel de significancia del $5 \%$. Se consideraron tendencias estadísticas cuando el valor de $\mathrm{p}$ fue $<0,05 \mathrm{o} \leq 0,1$.

Para el sistema LTSSPi, por problemas técnicos en el laboratorio, hubo necesidad de eliminar el perfil de AG de la leche del último tercio de lactancia y por esto el análisis se realizó con base en el tercio 1 y 2 .

\section{RESULTADOS Y DISCUSIÓN}

\section{Consumo de alimento}

El consumo diario de MS por animal en la mayoría de las fincas estuvo entre 8,23 y 13,42 kg MS (Cuadros 5 y 6) (entre 2,2 y $3,0 \%$ del peso vivo), valores que estuvieron de acuerdo con la proporción de suplemento consumido. Así, las fincas del sistema DPSSPi, que no recibían suplementación presentaron un menor consumo que las suplementadas. Por otra parte, las fincas Hatico del sistema LTSSPi y la finca Salsipuedes del sistema DPSSPi, presentaron un consumo bajo ocasionado por la leucaena, en Hatico se registró poca disponibilidad de este forraje con una oferta de tan solo $3,0 \mathrm{~kg}$ de forraje verde/animal/día, debido posiblemente a la edad del cultivo (más de diez años) en donde correspondió el muestreo, y en Salsipuedes se presentó rechazo de la leucaena por parte de los animales quedando el $60 \%$ de lo ofrecido, siendo la leucaena la principal leguminosa presente en los potreros.

La cantidad de alimento consumido está dentro de los rangos reportados para este tipo de animales; Mahecha et al. (2000), en un sistema silvopastoril, reportaron un consumo de forraje promedio de $9,5 \mathrm{~kg} /$ animal/ día, 7,7 kg de C. plectostachyus y 1,8 kg de leucaena, correspondiente a una proporción gramínea: leucaena de 81:19 y un consumo total de $13 \mathrm{~kg}$ MS/animal, incluyendo pastoreo más concentrado. En otro estudio, se reportaron consumos de forraje entre 8,25 y $11,8 \mathrm{~kg}$ de MS/animal y consumo total entre 9,75 - 13,3 kg de MS/animal en sistemas silvopastoriles de L. leucocephala cv. Cunningham asociada con P. máximum cv Tanzania, y para el sistema tradicional con $C$. plectostachyus un consumo diario de forraje de $3,63 \mathrm{~kg}$ de MS/animal y consumo total de 11,63 kg de MS/animal (Bacab-Pérez y Solorio-Sánchez, 2011). Igualmente, Restrepo (2013), 
reportó para un sistema silvopastoril de L. leucocephala asociada con Megathyrsus máximus y C. plectostachyus, un consumo de forraje de $8,47 \mathrm{~kg}$ de MS/animal y consumo total de $12,7 \mathrm{~kg}$ de MS/animal.

\section{Contenido de grasa en la leche}

En el sistema lechería tropical (LT), la dieta propia de cada finca y el número de parto, no afectaron el porcentaje de grasa de la leche $(\mathrm{p}>0,05)($ Cuadros 7 y 8$)$. Hubo una tendencia estadística $(p=0,09)$ al aumento en el contenido de grasa de la leche, con el tercio de lactancia, siendo el último tercio el que presentó la mayor cantidad de grasa (Cuadro 8). En el sistema lechería tropical sistema silvopastoril intensivo (LTSSPi), el contenido de grasa en leche no presentó diferencias entre fincas $(\mathrm{p}>0,05)$ (Cuadro 9), ni número de parto $(\mathrm{p}>0,05)$, pero presentó diferencia significativa por el tercio de lactancia $(p<0,05)$ (Cuadro 10), siendo el tercer tercio el que presentó la mayor cantidad de grasa (Cuadro 10).

En el sistema doble propósito (DP), el contenido de grasa en leche no fue afectado por la dieta dada en cada finca $(p>0,05)$, el número de parto $(p>0,05)$, ni el tercio de lactancia $(\mathrm{p}>0,05)$ (Cuadros 11 y 12).

En el sistema doble propósito sistema silvopastoril intensivo (DPSSPi), en las dos fincas evaluadas, el contenido de grasa en leche no se vio afectado por la dieta dada en cada finca $(\mathrm{p}>0,05)$, ni por el número de parto $(\mathrm{p}>0,05)$; pero sí por el tercio de lactancia $(\mathrm{p}<0,05)$, presentando el primer tercio el menor valor (Cuadros 13 y 14). El contenido de grasa en leche, en las fincas de los sistemas LT y LTSSPi (Cuadros 7 y 9), estuvo dentro de los valores normales al compararlo con los porcentajes de grasa en leche reportados por Calderón et al. (2006) para varias regiones de Colombia; sin embargo, las fincas del sistema DP presentaron valores promedio bajos $(2,70$ y 2,96) (Cuadro 11), mientras que las fincas del sistema DPSSPi presentaron valores promedio altos $(4,40$ y 4,99\%) (Cuadro 13). Los valores altos de grasa encontrados en el sistema DPSSPi estuvieron asociados con la menor producción de leche respecto a los valores promedios nacionales, esto hizo que ocurriera un efecto de concentración de los componentes de la leche. Existe una relación inversa entre la producción de leche y el porcentaje de constituyentes de la misma; cuando se produce más cantidad, los componentes disminuyen por tener un mayor factor de dilución (Campabadall, 1999; Prendiville et al., 2011). Esta explicación también aplicaría para la mayor concentración de grasa encontrada en el último tercio de lactancia.

En cuanto a los valores bajos de grasa en leche encontrados en el sistema DP, estos podrían deberse al efecto del amamantamiento de la cría. Se ha demostrado, que vacas que amamantan sus crías presentan menor porcentaje de grasa en leche para la venta, que aquellas que no lo hacen; por lo tanto, el porcentaje de grasa es mayor en la leche que consumen las crías, que en la leche para venta (Ojeda et al., 2001; Fröberg et al., 2007; Cozma et al., 2011). De acuerdo con lo anterior, las vacas del sistema doble propósito (DP) presentaron un porcentaje de grasa en la leche para la venta menor que el de las vacas de los sistemas LT y LTSSPi que no amamantaron la cría.

\section{Concentración de ácidos grasos en la leche y manejo alimenticio (finca)}

En el sistema lechería tropical, el ALC-c9t11 fue diferente entre fincas $(\mathrm{p}<0,05)$, siendo la finca Pradera la que presentó la menor proporción (Cuadro 7).

En este mismo sistema, el ATV fue diferente entre fincas $(\mathrm{p}<0,05)$, presentando la finca Pradera la menor proporción de ATV con respecto a las fincas Esperanza y San Felipe (Cuadro 7). El ácido linolénico presentó una mayor proporción en la leche de la finca San Felipe $(p<0,05)$. Se presentó interacción entre finca $x$ tercio de lactancia $(\mathrm{p}<0,05)$.

Los ácidos oleico y linoleico, no presentaron diferencias entre fincas $(p>0,05)$ (Cuadro 7).

La dieta propia de cada finca (finca) no afectó la proporción de AG saturados, insaturados, omega 6, aterogénicos, ni índice de aterogenicidad $(p>0,05)$ del mismo sistema (Cuadro 7).

En la leche de la finca Pradera, la proporción de ácidos grasos $\mathrm{n}-3$ fue menor $(\mathrm{p}<0,05)$ y la relación $\mathrm{n} 6 / \mathrm{n} 3$, fue mayor a las otras dos fincas $(\mathrm{p}<0,05)$ del mismo sistema. Igualmente, la finca Pradera presentó la menor proporción de ATV y de trans C18:1 (ATV + C18:1 t9) $(\mathrm{p}<0,05)$.

Los ácidos grasos omega 3, presentaron una marcada interacción de finca $\mathrm{x}$ tercio de lactancia $(\mathrm{p}<0,05)$ (Cuadro 8).

En el sistema lechería tropical sistema silvopastoril intensivo, los AG fueron cuantificados solo para el primero y segundo tercio. El ALC-c9t11 no fue diferente entre fincas $(\mathrm{p}<0,05)($ Cuadro 9). 
Cuadro 7. Contenido promedio de grasa y de ácidos grasos en la leche de vacas provenientes de las diferentes fincas (dietas) del sistema lechería tropical sin sistema silvopastoril intensivo de leucaena. Mayo-diciembre 2012. Colombia.

Table 7. Average content of fat and fatty acids in milk of cows from different farms (diets) in the tropical dairy production system without intensive silvopastoral system of leucaena. MayDecember 2012. Colombia.

\begin{tabular}{|c|c|c|c|c|c|c|c|}
\hline \multirow[t]{2}{*}{ Ácido graso } & \multicolumn{6}{|c|}{ Finca } & \multirow{2}{*}{$\frac{\text { valor de } p}{\text { Finca }}$} \\
\hline & \multicolumn{2}{|l|}{ Esperanza } & \multicolumn{2}{|l|}{ Pradera } & \multicolumn{2}{|c|}{ San Felipe } & \\
\hline \multirow[t]{2}{*}{$\%$ grasa } & 4,01 & & 3,48 & & 3,53 & & ns \\
\hline & \multicolumn{7}{|c|}{ g AG/100g AG totales } \\
\hline C6:0 & 1,52 & $\mathrm{a}$ & 1,31 & $\mathrm{ab}$ & 1,68 & $\mathrm{ac}$ & 0,0006 \\
\hline $\mathrm{C} 8: 0$ & 1,18 & a & 0,99 & $\mathrm{~b}$ & 1,15 & $\mathrm{a}$ & 0,0029 \\
\hline C10:0 & 2,24 & & 1,78 & & 2,06 & & ns \\
\hline C11:0 & 0,43 & & 0,39 & & 0,44 & & ns \\
\hline $\mathrm{C} 12: 0$ & 2,91 & & 2,53 & & 2,61 & & ns \\
\hline C13:0 & 0,23 & & 0,23 & & 0,25 & & ns \\
\hline $\mathrm{C} 14: 0+\mathrm{C} 14: 1$ cis & 15,56 & a & 13,56 & $\mathrm{~b}$ & 14,71 & $a b$ & 0,0311 \\
\hline $\mathrm{C} 15: 0+\mathrm{C} 15: 1$ cis & 2,48 & a & 2,22 & $a b$ & 2,73 & ac & 0,0154 \\
\hline $\mathrm{C} 16: 0$ & 21,60 & & 23,73 & & 21,98 & & ns \\
\hline $\mathrm{C} 16: 1 \mathrm{cis}$ & 1,33 & & 1,58 & & 1,44 & & ns \\
\hline $\mathrm{C} 17: 0$ & 0,90 & a & 0,78 & $\mathrm{a}$ & 1,10 & $\mathrm{~b}$ & $<0,0001$ \\
\hline $\mathrm{C} 17: 1$ cis 10 & 0,48 & $\mathrm{a}$ & 0,48 & $\mathrm{a}$ & 0,53 & $a b$ & 0,0294 \\
\hline C18:0 & 14,62 & & 16,61 & & 14,33 & & ns \\
\hline $\mathrm{C} 18: 1$ cis 9 & 21,79 & & 23,76 & & 22,17 & & ns \\
\hline C18:1 trans9 & 0,51 & & 0,59 & & 0,57 & & ns \\
\hline $\mathrm{C} 18: 1$ trans11 & 6,32 & $\mathrm{a}$ & 5,09 & $\mathrm{~b}$ & 6,34 & $\mathrm{a}$ & 0,0173 \\
\hline $\mathrm{C} 18: 2$ cis 9,12 & 2,10 & & 2,52 & & 1,97 & & ns \\
\hline $\mathrm{C} 18: 2$ cis $9, \operatorname{trans} 11$ & 2,09 & $\mathrm{a}$ & 1,28 & $\mathrm{~b}$ & 2,08 & $\mathrm{a}$ & 0,0165 \\
\hline C18:3 cis $6,9,12$ & 0,25 & & 0,21 & & 0,23 & & $\mathrm{~ns}$ \\
\hline C18:3 cis $9,12,15$ & 0,37 & a & 0,36 & $\mathrm{a}$ & 0,48 & $\mathrm{~b}$ & 0,0281 \\
\hline C20:0 & 0,32 & & 0,26 & & 0,32 & & ns \\
\hline \multirow[t]{2}{*}{$\mathrm{C} 22: 0$} & 0,13 & & 0,09 & & 0,15 & & ns \\
\hline & \multicolumn{7}{|c|}{ Sumatorias } \\
\hline Saturados & 64,10 & & 64,35 & & 64,31 & & ns \\
\hline Insaturados & 35,90 & & 35,65 & & 35,69 & & ns \\
\hline Monoinsaturados & 30,89 & & 31,04 & & 30,15 & & ns \\
\hline Poliinsaturados & 5,01 & & 4,61 & & 5,54 & & ns \\
\hline Omega 3 (n3) & 0,53 & a & 0,36 & $\mathrm{~b}$ & 0,57 & $\mathrm{a}$ & $<0,0001$ \\
\hline Omega 6 (n6) & 2,77 & & 2,77 & & 2,62 & & ns \\
\hline $\mathrm{n} 6 / \mathrm{n} 3$ & 5,70 & a & 7,97 & $\mathrm{~b}$ & 4,99 & $\mathrm{a}$ & 0,0180 \\
\hline $\mathrm{C} 10$ a C16 & 45,34 & & 44,34 & & 44,33 & & ns \\
\hline Trans C18:1 & 6,84 & $\mathrm{a}$ & 5,68 & $\mathrm{~b}$ & 6,89 & $\mathrm{a}$ & 0,0194 \\
\hline Aterogénicos C12, 14,16 & 40,12 & & 37,43 & & 39,14 & & $\mathrm{~ns}$ \\
\hline Índice de aterogenicidad & 2,42 & & 2,16 & & 2,36 & & ns \\
\hline
\end{tabular}

Valores con letras diferentes en la misma fila difieren significativamente entre tratamientos $(\mathrm{p}<0,05) /$ Values with different letters in the same row differ significantly between treatments $(\mathrm{p}<0.05)$.

El ATV no fue diferente entre fincas $(\mathrm{p}>0,05)$ (Cuadro 9), y hubo interacción entre finca $\mathrm{x}$ tercio de lactancia $(\mathrm{p}<0,05)$.
El ácido oleico presentó una mayor proporción en la leche de la finca Hatico $(\mathrm{p}<0,05)$, y se presentó interacción entre finca $x$ tercio de lactancia $(\mathrm{p}<0,05)$. 
Cuadro 8. Contenido promedio de grasa y de ácidos grasos en la leche de vacas provenientes del sistema lechería tropical sin sistema silvopastoril intensivo de leucaena, de acuerdo con el número de parto y tercio de lactancia. Mayo-diciembre 2012. Colombia.

Table 8. Average content of fat and fatty acids in milk of cows from the tropical dairy production system without intensive silvopastoral system of leucaena, according to the number of birth and stage of lactation. May-December. 2012. Colombia.

\begin{tabular}{|c|c|c|c|c|c|c|c|c|c|c|c|}
\hline \multirow[t]{2}{*}{ Ácido graso } & \multicolumn{2}{|c|}{ Parto } & \multicolumn{6}{|c|}{ Tercio de lactancia $(\mathrm{T})$} & \multicolumn{3}{|c|}{$\mathbf{p}<$} \\
\hline & 3 & 6 & 1 & & 2 & & 3 & & Parto & $\mathbf{T}$ & $\mathbf{F}^{*} \mathbf{T}$ \\
\hline \multirow[t]{2}{*}{$\%$ grasa } & 3,63 & 3,71 & 3,49 & $\mathrm{a}$ & 3,70 & $\mathrm{a}$ & 3,84 & $\mathrm{~b}$ & $\mathrm{~ns}$ & 0,0904 & ns \\
\hline & \multicolumn{11}{|c|}{ g AG/100g AG totales } \\
\hline C6:0 & 1,50 & 1,50 & 1,55 & $\mathrm{a}$ & 1,56 & $\mathrm{a}$ & 1,39 & $\mathrm{c}$ & ns & 0,0168 & 0,0005 \\
\hline C8:0 & 1,12 & 1,10 & 1,20 & a & 1,14 & $\mathrm{a}$ & 1,99 & $\mathrm{c}$ & $\mathrm{ns}$ & $<0,0001$ & ns \\
\hline C10:0 & 1,95 & 2,10 & 2,14 & $\mathrm{a}$ & 2,05 & $a b$ & 1,89 & $\mathrm{bc}$ & $\mathrm{ns}$ & 0,0064 & $\mathrm{~ns}$ \\
\hline C11:0 & 0,43 & 0,41 & 0,42 & $\mathrm{a}$ & 0,44 & $a b$ & 0,39 & ac & $\mathrm{ns}$ & 0,0327 & $\mathrm{~ns}$ \\
\hline C12:0 & 2,59 & 2,78 & 2,65 & & 2,76 & & 2,63 & & ns & ns & 0,0163 \\
\hline $\mathrm{C} 13: 0$ & 0,23 & 0,24 & 0,25 & & 0,23 & & 0,23 & & ns & ns & ns \\
\hline $\mathrm{C} 14: 0+\mathrm{C} 14: 1 \mathrm{cis}$ & 14,01 & 15,21 & 14,39 & & 14,93 & & 14,51 & & $\mathrm{~ns}$ & $\mathrm{~ns}$ & 0,0637 \\
\hline $\mathrm{C} 15: 0+\mathrm{C} 15: 1$ cis & 2,39 & 2,56 & 2,30 & $\mathrm{a}$ & 2,58 & $\mathrm{~b}$ & 2,54 & $\mathrm{~b}$ & $\mathrm{~ns}$ & 0,0003 & ns \\
\hline C16:0 & 21,67 & 23,20 & 21,33 & $\mathrm{a}$ & 22,63 & $\mathrm{~b}$ & 23,35 & $\mathrm{~b}$ & ns & $<0,0001$ & $<0,0001$ \\
\hline $\mathrm{C} 16: 1$ cis & 1,40 & 1,50 & 1,33 & $\mathrm{a}$ & 1,48 & $\mathrm{~b}$ & 1,54 & $\mathrm{~b}$ & ns & 0,0064 & 0,0708 \\
\hline $\mathrm{C} 17: 0$ & 0,93 & 0,92 & 1,00 & $\mathrm{a}$ & 0,90 & $\mathrm{~b}$ & 0,88 & $\mathrm{~b}$ & ns & 0,0002 & 0,0519 \\
\hline $\mathrm{C} 17: 1 \operatorname{cis} 10$ & 0,53 & 0,48 & 0,55 & $\mathrm{a}$ & 0,49 & $\mathrm{~b}$ & 0,47 & $\mathrm{~b}$ & ns & 0,0091 & \\
\hline C18:0 & 15,90 & 15,29 & 16,59 & $\mathrm{a}$ & 14,69 & $\mathrm{~b}$ & 14,29 & $\mathrm{~b}$ & $\mathrm{~ns}$ & $<0,0001$ & 0,0019 \\
\hline C18:1 cis9 & 24,35 & 20,80 & 22,88 & & 22,23 & & 22,61 & & ns & ns & ns \\
\hline C18:1 trans9 & 0,55 & 0,56 & 0,59 & $\mathrm{a}$ & 0,52 & $\mathrm{~b}$ & 0,56 & $a b$ & ns & 0,0085 & 0,0375 \\
\hline C18:1 trans11 & 5,61 & 6,21 & 6,12 & & 5,79 & & 5,83 & & ns & ns & 0,0179 \\
\hline $\mathrm{C} 18: 2$ cis 9,12 & 2,10 & 2,30 & 2,28 & & 2,19 & & 2,13 & & ns & ns & 0,0059 \\
\hline $\mathrm{C} 18: 2$ cis $9, \operatorname{trans} 11$ & 1,93 & 1,70 & 1,53 & $\mathrm{a}$ & 1,91 & $\mathrm{~b}$ & 2,01 & $\mathrm{~b}$ & $\mathrm{~ns}$ & 0,0001 & 0,0274 \\
\hline C18:3 cis $6,9,12$ & 0,26 & 0,20 & 0,16 & $\mathrm{a}$ & 0,24 & $\mathrm{~b}$ & 0,29 & $\mathrm{~b}$ & $\mathrm{~ns}$ & 0,0006 & ns \\
\hline C18:3 cis $9,12,15$ & 0,39 & 0,42 & 0,45 & & 0,40 & & 0,40 & & ns & ns & 0,0307 \\
\hline C20:0 & 0,30 & 0,30 & 0,28 & & 0,32 & & 0,30 & & $\mathrm{~ns}$ & ns & $\mathrm{ns}$ \\
\hline \multirow[t]{2}{*}{$\mathrm{C} 22: 0$} & 0,14 & 0,10 & 0,11 & & 0,13 & & 0,13 & & ns & $\mathrm{ns}$ & $\mathrm{ns}$ \\
\hline & \multicolumn{11}{|c|}{ Sumatorias } \\
\hline Saturados & 62,69 & 65,81 & 63,96 & & 64,45 & & 64,35 & & ns & $\mathrm{ns}$ & $\mathrm{ns}$ \\
\hline Insaturados & 37,31 & 34,19 & 36,04 & & 35,55 & & 35,65 & & ns & $\mathrm{ns}$ & ns \\
\hline Monoinsaturados & 32,53 & 28,86 & 31,65 & & 30,16 & & 30,26 & & ns & $\mathrm{ns}$ & $\mathrm{ns}$ \\
\hline Poliinsaturados & 4,78 & 5,33 & 4,39 & $\mathrm{a}$ & 5,39 & $\mathrm{~b}$ & 5,39 & $\mathrm{~b}$ & $\mathrm{~ns}$ & 0,0122 & 0,1707 \\
\hline Omega 3 (n3) & 0,51 & 0,41 & 0,42 & $\mathrm{a}$ & 0,47 & $\mathrm{~b}$ & 0,50 & $\mathrm{~b}$ & ns & 0,0244 & $<0,0001$ \\
\hline Omega 6 (n6) & 2,65 & 2,79 & 2,59 & & 2,81 & & 2,77 & & $\mathrm{~ns}$ & $\mathrm{~ns}$ & $\mathrm{~ns}$ \\
\hline $\mathrm{n} 6 / \mathrm{n} 3$ & 5,93 & 7,28 & 6,28 & & 7,02 & & 6,51 & & $\mathrm{~ns}$ & $\mathrm{~ns}$ & 0,0002 \\
\hline $\mathrm{C} 10$ a C16 & 43,33 & 46,01 & 43,50 & & 45,25 & & 45,26 & & ns & $\mathrm{ns}$ & 0,0081 \\
\hline Trans C18:1 & 6,18 & 6,76 & 6,72 & & 6,29 & & 6,40 & & $\mathrm{~ns}$ & $\mathrm{~ns}$ & 0,0079 \\
\hline Aterogénicos C12, 14,16 & 37,48 & 40,32 & 38,40 & & 40,31 & & 37,96 & & $\mathrm{~ns}$ & ns & $\mathrm{ns}$ \\
\hline Índice de aterogenicidad & 2,11 & 2,51 & 2,27 & & 2,41 & & 2,25 & & ns & ns & 0,0049 \\
\hline
\end{tabular}

$\mathrm{T}$ : tercio de lactancia, $\mathrm{F}^{*} \mathrm{~T}$ : finca * tercio. Valores con letras diferentes en la misma fila difieren significativamente entre tratamientos $(\mathrm{p}<0,05) / \mathrm{T}$ : stage of lactation, $\mathrm{F}^{*} \mathrm{~T}$ : farm * stage of lactation. Values with different letters in the same row differ significantly between treatments $(\mathrm{p}<0.05)$.

Los ácidos linoleico y linolénico, no presentaron diferencias entre fincas $(p>0,05)$ (Cuadro 9).
Los AG insaturados fueron diferentes entre fincas $(\mathrm{p}<0,05)$ y en la interacción finca $\mathrm{x}$ tercio de lactancia 
Cuadro 9. Contenido promedio de grasa y de ácidos grasos en la leche de vacas provenientes de las diferentes fincas (dietas) del sistema lechería tropical con sistema silvopastoril intensivo (LTSSPi) de leucaena. Mayo-diciembre 2012. Colombia.

Table 9. Average content of fat and fatty acids in milk of cows from different farms (diets) in the tropical dairy production system with intensive silvopastoral system (LTSSPi) of leucaena. May-December 2012. Colombia.

\begin{tabular}{|c|c|c|c|c|}
\hline \multirow[t]{2}{*}{ Ácido graso } & \multicolumn{3}{|c|}{ Finca } & \multirow{2}{*}{$\begin{array}{c}\text { Valor de } p \\
\text { Finca }\end{array}$} \\
\hline & Asturias & Hatico & Lucerna & \\
\hline \multirow{2}{*}{$\%$ grasa } & 3,16 & 3,43 & 3,45 & ns \\
\hline & \multicolumn{4}{|c|}{ g AG/100g AG totales } \\
\hline C6:0 & 1,44 & 1,32 & 1,40 & ns \\
\hline $\mathrm{C} 8: 0$ & 1,15 & 0,91 & 1,15 & ns \\
\hline C10:0 & $1,95 \mathrm{ab}$ & $1,48 \mathrm{~b}$ & 2,30 a & 0,0177 \\
\hline $\mathrm{C} 12: 0$ & $2,38 \mathrm{ab}$ & $1,96 \mathrm{~b}$ & 2,98 a & 0,0271 \\
\hline $\mathrm{C} 14: 0+\mathrm{C} 14: 1$ cis & $12,24 \mathrm{~b}$ & $11,11 \mathrm{~b}$ & $16,03 \mathrm{a}$ & 0,0148 \\
\hline $\mathrm{C} 15: 0+\mathrm{C} 15: 1 \mathrm{cis}$ & 2,16 & 1,80 & 1,90 & ns \\
\hline $\mathrm{C} 16: 0$ & 22,3 & 22,19 & 22,63 & $\mathrm{~ns}$ \\
\hline $\mathrm{C} 16: 1 \mathrm{cis}$ & 1,17 & 1,48 & 1,25 & ns \\
\hline $\mathrm{C} 17: 0$ & 1,14 a & $0,80 \mathrm{~b}$ & $0,88 \mathrm{~b}$ & 0,0314 \\
\hline C17:1 cis 10 & 0,57 & 0,44 & 0,46 & ns \\
\hline C18:0 & 18,53 & 17,01 & 15,68 & $\mathrm{~ns}$ \\
\hline $\mathrm{C} 18: 1$ cis9 & $23,91 \quad b$ & 27,28 a & $22,43 \mathrm{~b}$ & 0,0339 \\
\hline C18:1 trans 9 & $0,78 \mathrm{~b}$ & $1,08 \mathrm{a}$ & $0,62 \mathrm{~b}$ & 0,0025 \\
\hline $\mathrm{C} 18: 1$ trans 11 & 5,74 & 6,49 & 4,88 & ns \\
\hline $\mathrm{C} 18: 2$ cis 9,12 & 2,55 & 2,65 & 3,14 & $\mathrm{~ns}$ \\
\hline C18:2 cis9, trans 11 & 1,02 & 1,46 & 1,07 & $\mathrm{~ns}$ \\
\hline C18:3 cis6,9,12 & 0,31 & 0,26 & 0,26 & ns \\
\hline $\mathrm{C} 18: 3$ cis $9,12,15$ & 0,30 & 0,30 & 0,34 & $\mathrm{~ns}$ \\
\hline \multirow[t]{2}{*}{ C20:0 } & 0,37 & 0,38 & 0,31 & $\mathrm{~ns}$ \\
\hline & \multicolumn{4}{|c|}{ Sumatorias } \\
\hline Saturados & $63,67 \quad a$ & $58,46 \quad \mathrm{~b}$ & 65,45 a & 0,0189 \\
\hline Insaturados & $36,32 \mathrm{~b}$ & 41,53 a & $34,54 \mathrm{~b}$ & 0,0189 \\
\hline Monoinsaturados & $32,05 \mathrm{~b}$ & 36,85 a & $29,79 \mathrm{~b}$ & 0,0051 \\
\hline Poliinsaturados & 4,21 & 4,78 & 4,77 & ns \\
\hline Omega 3 (n-3) & 0,38 & 0,29 & 0,30 & $\mathrm{~ns}$ \\
\hline Omega $6(n-6)$ & 2,82 & 3,03 & 3,44 & $\mathrm{~ns}$ \\
\hline $\mathrm{n} 6 / \mathrm{n} 3$ & 8,14 & 11,39 & 11,16 & ns \\
\hline $\mathrm{C} 10$ a C16 & $41,14 \mathrm{~b}$ & $38,55 \mathrm{~b}$ & 46,51 a & 0,0187 \\
\hline Trans C18:1 & $6,36 \mathrm{ab}$ & 7,51 a & $5,51 \mathrm{~b}$ & 0,0351 \\
\hline Aterogénicos C12, 14,16 & $36,99 \mathrm{~b}$ & $35,07 \mathrm{~b}$ & 41,55 a & 0,0083 \\
\hline Índice de aterogenicidad & $2,12 \mathrm{a}$ & $1,69 \mathrm{~b}$ & $2,70 \quad \mathrm{a}$ & 0,0094 \\
\hline
\end{tabular}

Valores con letras diferentes en la misma fila difieren significativamente entre tratamientos $(\mathrm{p}<0,05)$

/ Values with different letters in the same row differ significantly between treatments $(\mathrm{p}<0.05)$.

$(\mathrm{p}<0,05)$, presentando la finca Hatico y el segundo tercio de lactancia, la mayor proporción. La proporción de AG monoinsaturados fue mayor en la finca Hatico $(\mathrm{p}<0,05)$, mientras que los AG poliinsaturados no presentaron diferencias entre fincas $(\mathrm{p}>0,05)$.

En este sistema, la proporción de AG omega 3, no fue diferente entre fincas $(\mathrm{p}>0,05)$.
Los ácidos grasos omega 6, no fueron diferentes entre fincas $(p>0,05)$, con interacción de finca $x$ tercio de lactancia $(\mathrm{p}<0,05)$, no obstante, no afectó la relación $\mathrm{n} 6 / \mathrm{n} 3$ en ninguno de los efectos evaluados $(\mathrm{p}>0,05)$.

La proporción de AG aterogénicos fue diferente entre fincas $(p<0,05)$, y hubo interacción finca $x$ tercio de lactancia $(\mathrm{p}<0,05)$, presentando el mayor valor la finca 
Lucerna y el primer tercio de lactancia. Sin embargo, el índice de aterogenicidad no presentó diferencias entre Lucerna y Asturias, siendo mayor en estas que en Hatico, y en el primer tercio de lactancia $(p<0,05)$ (Cuadro 9 y 10). En el sistema doble propósito, el
ALC-c9t11, no se afectó por la dieta dada en cada finca $(\mathrm{p}>0,05)$ (Cuadro 11) y la interacción finca $\mathrm{x}$ tercio de lactancia fue significativa $(\mathrm{p}<0,05)$ (Cuadro 12).

El ATV no fue diferente entre fincas $(p>0,05)$. El ácido linoleico presentó una mayor proporción en

Cuadro 10. Contenido promedio de grasa y de ácidos grasos en la leche de vacas provenientes del sistema lechería tropical con sistema silvopastoril intensivo (LTSSPi) de leucaena, de acuerdo al número de parto y tercio de lactancia. MayoDiciembre 2012. Colombia.

Table 10. Average content of fat and fatty acids in milk of cows from the tropical dairy production system with intensive silvopastoral system (LTSSPi) of leucaena, according to the number of birth and stage of lactation. May-December 2012. Colombia.

\begin{tabular}{|c|c|c|c|c|c|c|c|c|c|c|c|c|c|c|}
\hline \multirow[t]{2}{*}{ Ácido graso } & \multicolumn{7}{|c|}{ Parto } & \multicolumn{4}{|c|}{ Tercio de lactancia $(\mathbf{T})$} & \multicolumn{3}{|c|}{ Valor de $p$} \\
\hline & 3 & 4 & 5 & 6 & & 7 & & 1 & 2 & & 3 & Parto & $\mathbf{T}$ & $\mathbf{F} * \mathbf{T}$ \\
\hline \multirow[t]{2}{*}{$\%$ grasa } & 3,39 & 3,07 & 3,53 & 3,31 & & 3,43 & & $2,84 \mathrm{a}$ & 3,54 & $\mathrm{~b}$ & $3,66 \mathrm{~b}$ & ns & 0,0352 & ns \\
\hline & \multicolumn{14}{|c|}{ g AG/100g AG Totales } \\
\hline C6:0 & 1,35 & 0,85 & 1,30 & 1,24 & & 2,20 & & $1,71 \mathrm{a}$ & 1,06 & $\mathrm{~b}$ & & $\mathrm{~ns}$ & 0,0001 & 0,0103 \\
\hline C8:0 & 1,08 & 1,06 & 1,06 & 0,94 & & 1,21 & & $1,22 \mathrm{a}$ & 0,92 & $\mathrm{~b}$ & & $\mathrm{~ns}$ & 0,0087 & $\mathrm{~ns}$ \\
\hline C10:0 & 1,82 & 1,63 & 2,16 & 1,57 & & 2,37 & & $2,15 \mathrm{a}$ & 1,67 & $\mathrm{~b}$ & & ns & 0,0001 & 0,0113 \\
\hline C12:0 & 2,29 & 1,95 & 2,90 & 1,95 & & 3,11 & & $2,72 \mathrm{a}$ & 2,16 & $\mathrm{~b}$ & & ns & 0,0001 & 0,0063 \\
\hline $\mathrm{C} 14: 0+\mathrm{C} 14: 1$ cis & $12,18 \mathrm{~b}$ & b $\quad 9,76 \mathrm{~b}$ & $15,97 \mathrm{~b}$ & 11,31 & $\mathrm{~b}$ & 16,42 & $\mathrm{a}$ & $14,04 \mathrm{~b}$ & b 12,21 & $\mathrm{a}$ & & 0,0298 & 0,0001 & 0,0001 \\
\hline $\mathrm{C} 15: 0+\mathrm{C} 15: 1$ cis & 1,97 & 1,97 & 2,21 & 1,93 & & 1,69 & & 1,95 & 1,96 & & & ns & ns & 0,0403 \\
\hline C16:0 & 22,11 & 21,98 & 22,57 & 22,31 & & 22,91 & & 21,80 a & a 22,95 & $\mathrm{~b}$ & & ns & 0,0016 & 0,0037 \\
\hline $\mathrm{C} 16: 1$ cis & 1,05 & 1,39 & 1,63 & 1,18 & & 1,26 & & 1,22 & 1,38 & & & ns & ns & ns \\
\hline C17:0 & 0,99 & 1,03 & 0,81 & 1,01 & & 0,85 & & 0,93 & 0,95 & & & ns & ns & $\mathrm{ns}$ \\
\hline $\mathrm{C} 17: 1 \operatorname{cis} 10$ & 0,48 & 0,57 & 0,44 & 0,51 & & 0,45 & & 0,48 & 0,50 & & & ns & ns & ns \\
\hline C18:0 & 18,95 & 17,76 & 14,07 & 19,33 & & 15,25 & & 16,56 & 17,59 & & & ns & ns & ns \\
\hline C18: 1 cis9 & $25,03 \mathrm{~b}$ & 29,95 a & $23,54 \mathrm{~b}$ & 24,28 & $\mathrm{~b}$ & 19,9 & $\mathrm{~b}$ & 23,53 a & a 25,55 & $\mathrm{~b}$ & & 0,0372 & 0,0052 & 0,0425 \\
\hline C18:1 trans9 & 0,80 & 0,72 & 0,79 & 0,89 & & 0,93 & & $0,90 \mathrm{a}$ & 0,75 & $\mathrm{~b}$ & & ns & 0,0335 & ns \\
\hline C18:1 trans 11 & 4,92 & 4,87 & 5,61 & 6,23 & & 6,55 & & 5,07 a & 5,20 & $\mathrm{~b}$ & & ns & 0,0013 & 0,0262 \\
\hline $\mathrm{C} 18: 2$ cis 9,12 & 2,63 & 2,8 & 2,41 & 2,57 & & 3,5 & & 2,70 & 2,87 & & & ns & ns & 0,0029 \\
\hline $\mathrm{C} 18: 2$ cis 9 ,trans 11 & 0,86 & 1,00 & 1,44 & 1,22 & & 1,41 & & 1,23 & 1,14 & & & ns & ns & ns \\
\hline C18:3 cis6,9,12 & 0,34 & 0,39 & 0,22 & 0,26 & & 0,16 & & $0,22 \mathrm{a}$ & 0,33 & $\mathrm{~b}$ & & $\mathrm{~ns}$ & 0,0037 & 0,0084 \\
\hline $\mathrm{C} 18: 3$ cis $9,12,15$ & 0,26 & 0,23 & 0,32 & 0,31 & & 0,45 & & 0,31 & 0,32 & & & ns & ns & ns \\
\hline \multirow[t]{2}{*}{ C20:0 } & 0,43 & 0,34 & 0,29 & 0,39 & & 0,32 & & $0,32 \mathrm{a}$ & 0,39 & $\mathrm{~b}$ & & $\mathrm{~ns}$ & 0,0119 & $\mathrm{~ns}$ \\
\hline & \multicolumn{14}{|c|}{ Sumatorias } \\
\hline Saturados & 63,44 & 57,88 & 63,44 & 62,39 & & 65,48 & & 63,25 a & a 61,81 & $\mathrm{~b}$ & & $\mathrm{~ns}$ & 0,0414 & 0,0244 \\
\hline Insaturados & 36,55 & 42,11 & 36,55 & 37,60 & & 34,51 & & 36,74 a & a 38,18 & $\mathrm{~b}$ & & ns & 0,0414 & 0,0244 \\
\hline Monoinsaturados & 32,37 & 37,64 & 32,11 & 33,12 & & 29,23 & & 32,32 & 33,47 & & & $\mathrm{~ns}$ & ns & 0,0101 \\
\hline Poliinsaturados & 4,13 & 4,38 & 4,54 & 4,47 & & 5,43 & & 4,46 & 4,72 & & & ns & ns & 0,0016 \\
\hline Omega 3 (n-3) & 0,30 & 0,25 & 0,31 & 0,37 & & 0,37 & & 0,3 & 0,34 & & & ns & ns & ns \\
\hline Omega 6 (n-6) & 2,98 & 3,14 & 2,81 & 2,86 & & 3,69 & & $2,95 \mathrm{a}$ & 3,24 & $\mathrm{~b}$ & & ns & 0,0301 & 0,0009 \\
\hline n6/n3 & 11,55 & 12,86 & 8,75 & 8,97 & & 10,01 & & 10,10 & 10,76 & & & ns & ns & ns \\
\hline $\mathrm{C} 10$ a C16 & 40,76 a & $36,44 \mathrm{~b}$ & $46,12 \mathrm{a}$ & 39,37 & $\mathrm{~b}$ & 47,63 & $\mathrm{a}$ & 43,38 a & a 40,75 & $\mathrm{~b}$ & & 0,0502 & 0,0529 & 0,0015 \\
\hline Trans C18:1 & 5,77 & 5,64 & 6,38 & 7,10 & & 7,40 & & $6,97 \mathrm{a}$ & 5,95 & $\mathrm{~b}$ & & ns & 0,0013 & 0,0295 \\
\hline Aterogénicos C12, 14,16 & $36,68 \mathrm{~b}$ & $33,80 \mathrm{~b}$ & $41,26 \mathrm{a}$ & 35,57 & $\mathrm{~b}$ & 42,05 & $\mathrm{a}$ & 38,42 a & a 37,32 & $\mathrm{~b}$ & & 0,0289 & 0,0580 & 0,0003 \\
\hline Índice de aterogenicidad & 2,15 & 1,65 & 2,46 & 1,87 & & 2,71 & & $2,32 \mathrm{a}$ & 2,02 & $\mathrm{~b}$ & & $\mathrm{~ns}$ & 0,0003 & 0,0001 \\
\hline
\end{tabular}

T: tercio de lactancia, $\mathrm{F}^{*} \mathrm{~T}$ : finca * tercio. Valores con letras diferentes en la misma fila difieren significativamente entre tratamientos $(\mathrm{p}<0,05) / \mathrm{T}$ : stage of lactation, $\mathrm{F}^{*} \mathrm{~T}$ : farm * stage of lactation. Values with different letters in the same row differ significantly between treatments $(\mathrm{p}<0.05)$. 
Cuadro 11. Contenido promedio de grasa y de ácidos grasos en la leche de vacas provenientes de las diferentes fincas (dietas) del sistema doble propósito sin sistema silvopastoril intensivo de leucaena. Mayodiciembre 2012. Colombia.

Table 11. Average content of fat and fatty acids in milk of cows from different farms (diets) in the dual purpose production system without intensive silvopastoral system of leucaena. May-December 2012. Colombia.

\begin{tabular}{|c|c|c|c|}
\hline \multirow[t]{2}{*}{ Ácido graso } & \multicolumn{2}{|c|}{ Finca } & \multirow{2}{*}{$\begin{array}{c}\text { Valor de } \mathbf{p} \\
\text { Finca }\end{array}$} \\
\hline & Maracaibo & Vargas & \\
\hline$\%$ grasa & 2,70 & 2,96 & ns \\
\hline \multicolumn{4}{|c|}{ g AG/100g AG totales } \\
\hline C6:0 & 1,93 & 1,08 & ns \\
\hline C8:0 & 1,14 & 1,14 & ns \\
\hline C10:0 & 2,14 & 2,10 & ns \\
\hline C11:0 & 0,43 & 0,45 & ns \\
\hline C12:0 & 2,68 & 2,80 & ns \\
\hline C13:0 & 0,21 & 0,27 & $\mathrm{~ns}$ \\
\hline $\mathrm{C} 14: 0+\mathrm{C} 14: 1 \mathrm{cis}$ & 15,08 & 15,41 & ns \\
\hline $\mathrm{C} 15: 0+\mathrm{C} 15: 1$ cis & 2,59 & 2,77 & ns \\
\hline $\mathrm{C} 16: 0$ & 21,36 & 23,31 & ns \\
\hline $\mathrm{C} 16: 1$ cis & 1,47 & 1,74 & ns \\
\hline $\mathrm{C} 17: 0$ & 1,05 & 0,98 & ns \\
\hline $\mathrm{C} 17: 1 \operatorname{cis} 10$ & 0,56 & 0,60 & ns \\
\hline C18:0 & 14,30 & 13,50 & ns \\
\hline $\mathrm{C} 18: 1$ cis 9 & 22,78 & 22,49 & ns \\
\hline C18:1 trans9 & 0,66 & 0,62 & ns \\
\hline C18:1 trans 11 & 6,50 & 6,26 & ns \\
\hline C18:2 cis 9,12 & $1,92 \mathrm{a}$ & $1,40 \mathrm{~b}$ & 0,0249 \\
\hline $\mathrm{C} 18: 2$ cis 9 ,trans 11 & 1,98 & 2,22 & ns \\
\hline $\mathrm{C} 18: 2$ trans 9,12 & 0,35 & 0,42 & ns \\
\hline C18:3 cis6,9,12 & 0,17 & 0,20 & ns \\
\hline C18:3 cis $9,12,15$ & 0,44 & 0,34 & ns \\
\hline $\mathrm{C} 20: 0$ & 0,27 & 0,23 & ns \\
\hline \multicolumn{4}{|c|}{ Sumatorias } \\
\hline Saturados & 63,03 & 63,63 & ns \\
\hline Insaturados & 36,97 & 36,37 & ns \\
\hline Monoinsaturados & 31,96 & 31,76 & ns \\
\hline Poliinsaturados & 5,01 & 4,61 & ns \\
\hline Omega 3 (n-3) & $0,55 \mathrm{a}$ & $0,34 \mathrm{~b}$ & 0,0099 \\
\hline Omega 6 (n-6) & 2,40 & 1,99 & ns \\
\hline n6/n3 & 4,65 & 5,81 & ns \\
\hline $\mathrm{C} 10$ a C16 & 44,68 & 45,96 & ns \\
\hline Trans C18:1 & 7,16 & 6,90 & ns \\
\hline Aterogénicos C12, 14,16 & 39,11 & 41,51 & ns \\
\hline Índice de aterogenicidad & 2,32 & 2,46 & ns \\
\hline
\end{tabular}

Valores con letras diferentes en la misma fila difieren significativamente entre tratamientos $(\mathrm{p}<0,05) /$ Values with different letters in the same row differ significantly between treatments $(\mathrm{p}<0.05)$ la leche de la finca Maracaibo $(\mathrm{p}<0,05)$ y se presentó interacción entre finca $\mathrm{x}$ tercio de lactancia $(\mathrm{p}<0,05)$. Los ácidos oleico y linolénico, no presentaron diferencias entre fincas ( $\mathrm{p}>0,05)$. La proporción de AG saturados, insaturados, omega 6, n6/n3, C10 a C16, trans C18:1, aterogénicos e índice de aterogenicidad, no fueron afectados por la finca $(p>0,05)$. Solo los ácidos omega 3 presentaron diferencias entre fincas $(\mathrm{p}<0,05)$, siendo la finca Maracaibo la que presentó la mayor proporción con respecto a la finca Vargas que solo registró linolénico en la leche y los demás n3 no los registró, aunque la relación n6/n3 no se vio afectada $(\mathrm{p}>0,05)$.

En el sistema doble propósito sistema silvopastoril intensivo, el ALC-c9t11 no fue diferente entre fincas $(\mathrm{p}>0,05)$ (Cuadro 13). El ATV fue diferente entre fincas $(\mathrm{p}<0,05)$ (Cuadro 13), siendo la finca Pradera la que presentó la mayor proporción. Los ácidos oleico, linoleico y linolénico de la leche, no presentaron diferencias entre fincas ( $p>0,05)$ (Cuadro 13). La proporción de AG saturados, insaturados, omega 6 , aterogénicos, índice de aterogenicidad, no fueron afectados por la finca $(\mathrm{p}>0,05)$.

La proporción de trans C18:1 fue diferente entre fincas $(\mathrm{p}<0,05)$, siendo mayor en la finca Pradera.

La concentración de ALC-c9t11 en la grasa de la leche presentó valores entre 1,28\% y 2,22\%, y la proporción de ATV estuvo entre 4,40 y 6,50\%. Los valores de ALC-c9t11 se encontraron dentro del rango reportado por Khanal y Olson (2004) de 0,2\%-3,7\%. No obstante, algunos de los valores de ALC-c9t11 fueron superiores al valor promedio reportado por Rico et al. (2007) de 1,35\% o Vargas et al. (2013) de 1,0 a $1,2 \%$, en animales bajo pastoreo en Sabana de Bogotá. También fueron más altos en comparación con los valores reportados con animales estabulados, alimentados con forrajes conservados en TMR (ración total mezclada) y con suplementación lipídica $(0,31$ y $0,61 \%)$; igual situación se presentó con los valores de ATV, los cuales fueron bajos $(0,62$ y $1,65 \%)$ para los grupos control (sin adición de aceite) en estos mismos trabajos (Ferlay et al., 2013; Boerman y Lock, 2014; Saliba et al., 2014; Vargas-Bello-Pérez et al., 2015). Los resultados obtenidos en este estudio para ATV fueron superiores a los reportados por Vargas et al. (2013), los cuales se encontraron entre 2,6 a 3,3\%; pero fueron similares a los encontrados por Angulo et al. (2012a), quienes encontraron valores de 1,6 a $1,8 \%$ para ALC-c9t11 y de 4,7 a $6,9 \%$ para ATV, 
Cuadro 12. Contenido promedio de grasa y de ácidos grasos en la leche de vacas provenientes del sistema doble propósito sin sistema silvopastoril intensivo de leucaena, de acuerdo con el número de parto y tercio de lactancia. Mayo-diciembre 2012. Colombia.

Table 12. Average content of fat and milk fatty acids in milk of cows from the dual purpose production system without intensive silvopastoral system of leucaena, according to the number of birth and stage of lactation. May-December. 2012. Colombia.

\begin{tabular}{|c|c|c|c|c|c|c|c|c|c|c|}
\hline \multirow[t]{2}{*}{ Ácido graso } & \multicolumn{4}{|c|}{ Parto } & \multicolumn{3}{|c|}{ Tercio lactancia } & \multicolumn{3}{|c|}{ Valor de $p$} \\
\hline & 2 & 3 & 4 & 5 & 1 & 2 & 3 & Parto & Tercio & $\mathbf{F}^{*} \mathbf{T}$ \\
\hline$\%$ grasa & 2,88 & 2,65 & 3,16 & 2,63 & 2,4 & 2,94 & 3,15 & ns & ns & ns \\
\hline \multicolumn{11}{|c|}{ g AG/100g AG totales } \\
\hline C6:0 & 2,06 & 0,77 & 1,45 & 1,74 & 1,6 & 1,89 & 1,02 & ns & ns & ns \\
\hline C8:0 & 1,15 & 1,33 & 1,07 & 1,01 & $1,38 \mathrm{a}$ & $0,96 \mathrm{~b}$ & $1,08 \mathrm{~b}$ & ns & 0,0005 & 0,0422 \\
\hline C10:0 & $2,27 \mathrm{a}$ & $2,33 \mathrm{a}$ & $2,02 \mathrm{ab}$ & $1,87 \mathrm{~b}$ & $2,41 \mathrm{a}$ & $1,83 \mathrm{~b}$ & $2,12 \mathrm{~b}$ & 0,064 & 0,0013 & ns \\
\hline $\mathrm{C} 11: 0$ & 0,43 & 0,43 & 0,46 & 0,43 & 0,46 & 0,39 & 0,46 & ns & ns & 0,0106 \\
\hline $\mathrm{C} 12: 0$ & $2,95 \mathrm{a}$ & $2,94 \mathrm{a}$ & $2,68 \mathrm{ab}$ & $2,41 \mathrm{~b}$ & 2,99 a & $2,42 \mathrm{~b}$ & $2,82 \mathrm{a}$ & 0,0127 & 0,0009 & 0,0787 \\
\hline C13:0 & 0,27 & 0,28 & 0,20 & 0,21 & 0,27 & 0,20 & 0,26 & ns & ns & ns \\
\hline $\mathrm{C} 14: 0+\mathrm{C} 14: 1 \mathrm{cis}$ & 15,84 & 14,50 & 15,28 & 15,39 & 15,71 & 14,79 & 15,25 & ns & ns & $\mathrm{ns}$ \\
\hline $\mathrm{C} 15: 0+\mathrm{C} 15: 1 \mathrm{cis}$ & 2,65 & 2,59 & 2,74 & 2,74 & 2,55 & 2,63 & 2,86 & ns & ns & 0,0005 \\
\hline C16:0 & 22,98 & 21,45 & 22,43 & 22,50 & 21,82 & 22,52 & 22,67 & ns & ns & ns \\
\hline $\mathrm{C} 16: 1$ cis & 1,52 & 1,60 & 1,39 & 1,76 & 1,47 & 1,64 & 1,71 & ns & ns & 0,0315 \\
\hline $\mathrm{C} 17: 0$ & 0,99 & 1,09 & 2,00 & 0,98 & $1,11 \mathrm{a}$ & $0,88 \mathrm{~b}$ & $1,05 \mathrm{a}$ & ns & 0,0003 & 0,0358 \\
\hline $\mathrm{C} 17: 1 \operatorname{cis} 10$ & 0,51 & 0,65 & 0,55 & 0,60 & $0,66 \mathrm{a}$ & $0,41 \mathrm{~b}$ & $0,59 \mathrm{ab}$ & ns & 0,008 & 0,0055 \\
\hline $\mathrm{C} 18: 0$ & 13,99 & 14,69 & 13,47 & 13,45 & 14,61 & 13,43 & 13,66 & ns & ns & 0,0312 \\
\hline C18:1 cis9 & 20,56 & 23,16 & 23,20 & 23,61 & 21,91 & 23,54 & 23,45 & ns & ns & ns \\
\hline C18:1 trans9 & 0,62 & 0,67 & 0,61 & 0,67 & 0,70 & 0,59 & 0,64 & ns & ns & 0,0037 \\
\hline C18:1 trans11 & 6,49 & 6,38 & 6,42 & 6,23 & 6,23 & 6,48 & 6,43 & ns & ns & 0,0055 \\
\hline $\mathrm{C} 18: 2$ cis 9,12 & $1,48 \mathrm{a}$ & $2,11 \mathrm{~b}$ & $1,53 \mathrm{a}$ & $1,52 \mathrm{a}$ & 1,79 & 1,48 & 1,71 & 0,0258 & ns & 0,0333 \\
\hline $\mathrm{C} 18: 2$ cis9, trans11 & 2,00 & 2,19 & 2,15 & 2,71 & $1,65 \mathrm{a}$ & $2,37 \mathrm{~b}$ & $2,26 \mathrm{~b}$ & ns & 0,0034 & 0,0207 \\
\hline $\mathrm{C} 18: 2$ trans 9,12 & 0,35 & 0,35 & 0,45 & 0,40 & 0,33 & 0,40 & 0,44 & ns & ns & $\mathrm{ns}$ \\
\hline C18:3 cis6,9,12 & 0,20 & 0,19 & 0,19 & 0,16 & $0,14 \mathrm{a}$ & $0,28 \mathrm{~b}$ & $0,13 \mathrm{a}$ & ns & 0,0002 & ns \\
\hline $\mathrm{C} 18: 3$ cis $9,12,15$ & 0,40 & 0,45 & 0,38 & 0,35 & 0,40 & 0,36 & 0,42 & ns & ns & ns \\
\hline \multirow[t]{2}{*}{$\mathrm{C} 20: 0$} & 0,25 & 0,29 & 0,19 & 0,25 & 0,26 & 0,23 & 0,25 & ns & ns & ns \\
\hline & \multicolumn{10}{|c|}{ Sumatorias } \\
\hline Saturados & 65,77 & 62,09 & 62,89 & 62,56 & 64,80 & 62,08 & 63,11 & ns & ns & ns \\
\hline Insaturados & 34,23 & 37,91 & 37,11 & 37,44 & 35,20 & 37,92 & 36,89 & ns & ns & ns \\
\hline Monoinsaturados & 29,73 & 32,55 & 32,30 & 32,87 & 30,99 & 32,78 & 31,81 & ns & ns & ns \\
\hline Poliinsaturados & 4,51 & 5,36 & 4,81 & 4,57 & 4,21 & 5,14 & 5,08 & ns & ns & 0,0421 \\
\hline Omega 3 (n-3) & 0,45 & 0,53 & 0,45 & 0,36 & 0,40 & 0,42 & 0,52 & ns & ns & ns \\
\hline Omega 6 (n-6) & 1,96 & 2,63 & 2,12 & 2,07 & 2,13 & 2,21 & 2,25 & ns & ns & ns \\
\hline $\mathrm{n} 6 / \mathrm{n} 3$ & 4,51 & 5,58 & 5,21 & 5,62 & 5,68 & 5,41 & 5,60 & ns & ns & ns \\
\hline $\mathrm{C} 10$ a C16 & 47,60 & 46,28 & 45,38 & 45,02 & 46,15 & 44,61 & 45,20 & ns & ns & ns \\
\hline Trans C18:1 & 7,10 & 7,09 & 7,03 & 6,89 & 6,95 & 7,06 & 7,08 & ns & ns & $\mathrm{n}$ \\
\hline $\begin{array}{l}\text { Aterogénicos C12, } \\
14,16 \\
\text { Índice de }\end{array}$ & 41,73 & 38,88 & 40,36 & 40,27 & 40,50 & 39,70 & 40,72 & ns & ns & ns \\
\hline aterogenicidad & 2,65 & 2,20 & 2,31 & 2,39 & 2,53 & 2,25 & 2,39 & ns & ns & ns \\
\hline
\end{tabular}

$\mathrm{F}^{*} \mathrm{~T}$ : finca * tercio de lactancia. Valores con letras diferentes en la misma fila difieren significativamente entre tratamientos $(\mathrm{p}<0,05) /$ $\mathrm{F}^{*} \mathrm{~T}$ : farm * stage of lactation. Values with different letters in the same row differ significantly between treatments $(\mathrm{p}<0.05)$.

alimentando con TMR e incluyendo diferentes tipos de fuentes de AG poliinsaturados más alga marina.
Los altos valores obtenidos de ALC-c9t11 y ATV en la leche podrían estar relacionados con la utilización 
Cuadro 13. Contenido promedio de grasa y de ácidos grasos en la leche de vacas provenientes de diferentes fincas (dietas) del sistema doble propósito con sistema silvopastoril intensivo (DPSSPi) de leucaena. Mayo - diciembre 2012. Colombia.

Table 13. Average content of fat and fatty acids in milk of cows from different farms (diets) in the dual purpose production system with intensive silvopastoral system (DPSSPi) of leucaena. MayDecember 2012. Colombia.

\begin{tabular}{|c|c|c|c|}
\hline \multirow[t]{2}{*}{ Ácido graso } & \multicolumn{2}{|c|}{ Finca } & \multirow{2}{*}{$\begin{array}{c}\text { Valor de p } \\
\text { Finca } \\
\end{array}$} \\
\hline & Pradera & Salsipuedes & \\
\hline \multirow[t]{2}{*}{$\%$ grasa } & 4,40 & 4,99 & ns \\
\hline & \multicolumn{3}{|c|}{ g AG/100g AG totales } \\
\hline C6:0 & 1,46 & 1,36 & ns \\
\hline $\mathrm{C} 8: 0$ & 1,08 & 0,96 & ns \\
\hline C10:0 & 1,87 & 1,87 & ns \\
\hline C11:0 & 0,33 & 0,39 & ns \\
\hline C12:0 & 2,42 & 2,55 & ns \\
\hline C13:0 & 0,21 & 0,23 & ns \\
\hline $\mathrm{C} 14: 0+\mathrm{C} 14: 1$ cis & 14,87 & 16,42 & ns \\
\hline $\mathrm{C} 15: 0+\mathrm{C} 15: 1 \mathrm{cis}$ & 2,58 & 3,07 & ns \\
\hline C16:0 & 24,21 & 24,13 & ns \\
\hline $\mathrm{C} 16: 1$ cis & 1,42 & 1,43 & ns \\
\hline $\mathrm{C} 17: 0$ & 1,13 & 1,18 & ns \\
\hline $\mathrm{C} 17: 1$ cis 10 & 0,52 & 0,58 & ns \\
\hline C18:0 & 14,58 & 14,18 & ns \\
\hline C18: 1 cis 9 & 21,56 & 22,37 & ns \\
\hline C18:1 trans9 & 0,55 & 0,41 & ns \\
\hline $\mathrm{C} 18: 1$ trans 11 & 5,89 a & $4,40 \mathrm{~b}$ & 0,0227 \\
\hline C18:2 cis9,12 & 1,01 & 1,25 & ns \\
\hline C18:2 cis9,trans 11 & 2,05 & 1,43 & ns \\
\hline $\mathrm{C} 18: 2$ trans 9,12 & 0,28 & 0,15 & ns \\
\hline C18:3 cis6,9,12 & 0,26 & 0,25 & ns \\
\hline $\mathrm{C} 18: 3$ cis $9,12,15$ & 0,43 & 0,41 & ns \\
\hline C20:0 & 0,36 & 0,31 & ns \\
\hline C22:0 & 0,19 & 0,16 & ns \\
\hline \multirow[t]{2}{*}{$\mathrm{C} 24: 0$} & 0,10 & 0,13 & ns \\
\hline & \multicolumn{3}{|c|}{ Sumatorias } \\
\hline Saturados & 65,36 & 66,95 & ns \\
\hline Insaturados & 34,64 & 33,05 & ns \\
\hline Monoinsaturados & 30,03 & 29,29 & ns \\
\hline Poliinsaturados & 4,61 & 3,76 & ns \\
\hline Omega 3 (n-3) & 0,66 & 0,63 & ns \\
\hline Omega 6 (n-6) & 1,79 & 1,69 & ns \\
\hline $\mathrm{n} 6 / \mathrm{n} 3$ & 2,68 & 2,88 & ns \\
\hline $\mathrm{C} 10$ a C16 & 46,22 & 47,65 & ns \\
\hline Trans C18:1 & 6,45 a & $4,84 \mathrm{~b}$ & 0,0234 \\
\hline Aterogénicos $\mathrm{C} 12$, & & & \\
\hline 14,16 & 41,52 & 43,08 & ns \\
\hline Índice de & & & \\
\hline aterogenicidad & 2,53 & 2,89 & ns \\
\hline
\end{tabular}

Valores con letras diferentes en la misma fila difieren significativamente entre tratamientos $(\mathrm{p}<0,05)$ / Values with different letters in the same row differ significantly between treatments $(\mathrm{p}<0.05)$. de dietas con alta proporción de pasto en todas las fincas evaluadas. Además, podría estar relacionado, con la alta proporción de AG poliinsaturados en el concentrado utilizado. El pasto es una fuente rica de AG poliinsaturados, principalmente linolénico (18: $3 n-3)$, que se contribuye como precursor de ALCc9t11 y de C18:1 trans-11 (Chilliard et al., 2007). En todos los forrajes de los sistemas evaluados, el AG que estuvo en mayor proporción fue el ácido linolénico con valores entre 34,8 y $47,3 \%$, valor similar al reportado por Vargas et al. (2013) de 41\%, para pasto kikuyo a edad de rebrote de 45 días, pero menor al reportado por Elgersma et al. (2006) de 50-70\%. Además, se ha comprobado que la suplementación con ácido linoleico es efectiva para aumentar ALC-c9t11 (Hervás et al., 2006; Shingfield et al., 2006; Rego et al., 2009; Angulo et al., 2012a), esto podría ser debido a que la suplementación con ácido linoleico favorece la formación de ATV y ALC-c9t11, que llega de rumen a glándula mamaria e incrementa los niveles de este último ácido graso, a partir del ATV. El ácido linoleico se presentó en mayor proporción en los suplementos, a excepción del suplemento utilizado en Hatico que presentó mayor proporción de ácido oleico.

Los forrajes utilizados, fuente de linolénico y linoleico, constituyen la base alimenticia; no obstante, en los sistemas LT, LTSSPi y DP se utilizó suplementación con alimentos concentrados, consumiendo una proporción forraje:concentrado de 70:30 en LT y LTSSPi y de 80:20 en DP. Estos concentrados, altos en MS y con mayor porcentaje de grasa que los forrajes, hacen el mayor aporte de la grasa consumida. Se estimó que del consumo total diario de grasa, el concentrado estuvo aportando entre el 60 y $84 \%$ de la grasa en estos sistemas (Cuadros 5 y 6). Por lo tanto, las diferencias presentadas en la proporción de ATV, ALC-c9t11, omega 3, relación n6/n3 y trans C18:1 en el sistema $\mathrm{LT}$, en la proporción de insaturados, monoinsaturados, trans C18:1, aterogénicos e índice de aterogenicidad en el sistema LTSSPi, y de ácido linoleico en la leche del DP, fueron determinadas por las diferencias en la cantidad del AG precursor existente en el suplemento consumido en cada finca.

En el sistema LT el porcentaje estimado de ácido linoleico del concentrado, estuvo entre el 27,06 y $30,78 \%$ del total de grasa consumida, proporción mayor a la de los ácidos oleico y linolénico de forraje y del concentrado. Asimismo, el ácido linoléico 
Cuadro 14. Contenido promedio de grasa y de ácidos grasos en la leche de vacas provenientes del sistema doble propósito con sistema silvopastoril intensivo (DPSSPi) de leucaena, de acuerdo con el número de parto y tercio de lactancia. Mayo-diciembre 2012. Colombia.

Table 14. Average content of fat and fatty acids in milk of cows from the dual purpose production system with intensive silvopastoral system (DPSSPi) of leucaena, according to the number of birth and stage of lactation. May-December. 2012. Colombia.

\begin{tabular}{|c|c|c|c|c|c|c|c|c|c|c|c|c|c|c|}
\hline \multirow[t]{2}{*}{ Ácido graso } & \multicolumn{5}{|c|}{ Parto } & \multicolumn{6}{|c|}{ Tercio lactancia } & \multicolumn{3}{|c|}{ Valor de $p$} \\
\hline & 1 & 3 & 4 & 5 & 6 & 1 & & 2 & & 3 & & Parto & $\begin{array}{c}\text { Tercio } \\
\text { lac }\end{array}$ & $\begin{array}{c}\text { Finca * } \\
\text { tercio }\end{array}$ \\
\hline \multirow[t]{2}{*}{$\%$ grasa } & 4,41 & 4,8 & 4,55 & 4,97 & 4,74 & 3,83 & $\mathrm{a}$ & 5,1 & $\mathrm{~b}$ & 5,15 & $\mathrm{~b}$ & ns & $<0,0001$ & ns \\
\hline & \multicolumn{14}{|c|}{ g AG/100g AG totales } \\
\hline C6:0 & 1,41 & 1,46 & 1,38 & 1,37 & 1,43 & 1,41 & & 1,42 & & 1,40 & & $\mathrm{~ns}$ & ns & ns \\
\hline C8:0 & 1,02 & 1,12 & 1,97 & 1,01 & 1,99 & 1,06 & $\mathrm{a}$ & 1,14 & $\mathrm{a}$ & 0,88 & $\mathrm{~b}$ & ns & 0,0006 & 0,0478 \\
\hline C10:0 & 1,83 & 2,04 & 1,77 & 1,87 & 1,84 & 2,04 & $\mathrm{a}$ & 2,08 & $\mathrm{a}$ & 1,48 & $\mathrm{~b}$ & ns & 0,0014 & 0,068 \\
\hline $\mathrm{C} 11: 0$ & 0,38 & 0,37 & 0,33 & 0,36 & 0,38 & 0,40 & a & 0,40 & $\mathrm{a}$ & 0,29 & $\mathrm{~b}$ & ns & 0,0031 & ns \\
\hline C12:0 & 2,46 & 2,63 & 2,40 & 2,51 & 2,43 & 2,65 & $\mathrm{a}$ & 2,81 & $\mathrm{a}$ & 2,00 & $\mathrm{~b}$ & ns & 0,0025 & ns \\
\hline C13:0 & 0,23 & 0,22 & 0,21 & 0,23 & 0,19 & 0,23 & & 0,23 & & 0,19 & & $\mathrm{~ns}$ & $\mathrm{~ns}$ & $\mathrm{~ns}$ \\
\hline $\mathrm{C} 14: 0+\mathrm{C} 14: 1 \mathrm{cis}$ & 15,54 & 15,64 & 15,97 & 14,84 & 16,23 & 15,86 & $a b$ & 16,70 & $\mathrm{a}$ & 14,37 & $\mathrm{~b}$ & $\mathrm{~ns}$ & 0,0483 & ns \\
\hline $\mathrm{C} 15: 0+\mathrm{C} 15: 1 \mathrm{cis}$ & 2,81 & 2,80 & 2,78 & 2,71 & 3,03 & 2,73 & & 2,83 & & 2,93 & & ns & $\mathrm{ns}$ & 0,0943 \\
\hline C16:0 & 21,84 & 24,97 & 23,39 & 25,04 & 25,62 & 23,61 & & 25,65 & & 23,29 & & ns & ns & ns \\
\hline $\mathrm{C} 16: 1 \mathrm{cis}$ & 1,12 & 1,52 & 1,51 & 1,41 & 1,56 & 1,45 & & 1,51 & & 1,31 & & ns & ns & 0,000028 \\
\hline $\mathrm{C} 17: 0$ & 1,21 & 1,15 & 1,55 & 1,19 & 1,07 & 1,10 & $\mathrm{a}$ & 1,09 & $\mathrm{a}$ & 1,28 & $\mathrm{~b}$ & $\mathrm{~ns}$ & $<0,0001$ & ns \\
\hline $\mathrm{C} 17: 1 \operatorname{cis} 10$ & 0,47 & 0,55 & 0,56 & 0,57 & 0,53 & 0,56 & & 0,54 & & 0,56 & & $\mathrm{~ns}$ & ns & $\mathrm{ns}$ \\
\hline C18:0 & 16,15 & 14,38 & 14,07 & 14,38 & 12,91 & 14,23 & $a b$ & 13,06 & $\mathrm{a}$ & 15,85 & $\mathrm{~b}$ & $\mathrm{~ns}$ & 0,0122 & 0,0066 \\
\hline C18:1 cis9 & 22,46 & 21,03 & 22,94 & 22,12 & 21,27 & 22,06 & & 20,67 & & 23,17 & & ns & ns & $\mathrm{ns}$ \\
\hline C18:1 trans9 & 0,50 & 0,55 & 0,43 & 0,47 & 0,46 & 0,51 & & 0,50 & & 0,44 & & $\mathrm{~ns}$ & ns & 0,0003 \\
\hline C18:1 trans11 & 5,68 & 5,20 & 4,76 & 4,95 & 5,14 & 5,39 & & 4,90 & & 5,15 & & $\mathrm{~ns}$ & $\mathrm{~ns}$ & $\mathrm{~ns}$ \\
\hline $\mathrm{C} 18: 2$ cis9,12 & 1,27 & 1,03 & 1,26 & 1,14 & 0,96 & 1,15 & & 1,05 & & 1,18 & & ns & ns & $\mathrm{ns}$ \\
\hline C18:2 cis9, trans 11 & 1,67 & 1,64 & 1,75 & 1,73 & 1,91 & 1,80 & & 1,73 & & 1,70 & & $\mathrm{~ns}$ & $\mathrm{~ns}$ & 0,0943 \\
\hline $\mathrm{C} 18: 2$ trans 9,12 & 0,15 & 0,30 & 0,17 & 0,23 & 0,23 & 0,19 & & 0,25 & & 0,22 & & ns & $\mathrm{ns}$ & $\mathrm{ns}$ \\
\hline C18:3 cis6,9,12 & 0,23 & 0,26 & 0,30 & 0,23 & 0,25 & 0,22 & & 0,26 & & 0,27 & & ns & ns & 0,0101 \\
\hline C18:3 cis $9,12,15$ & 0,46 & 0,40 & 0,49 & 0,40 & 0,35 & 0,41 & & 0,40 & & 0,46 & & $\mathrm{~ns}$ & $\mathrm{~ns}$ & ns \\
\hline C20:0 & 0,37 & 0,31 & 0,34 & 0,34 & 0,31 & 0,27 & $\mathrm{a}$ & 0,32 & $\mathrm{a}$ & 0,41 & $\mathrm{~b}$ & ns & 0,0088 & 0,0129 \\
\hline C22:0 & 0,17 & 0,18 & 0,19 & 0,19 & 0,15 & 0,17 & $a b$ & 0,15 & $\mathrm{a}$ & 0,21 & $\mathrm{~b}$ & $\mathrm{~ns}$ & 0,0362 & $\mathrm{~ns}$ \\
\hline \multirow[t]{2}{*}{$\mathrm{C} 24: 0$} & 0,11 & 0,11 & 0,11 & 0,12 & 0,11 & 0,10 & & 0,11 & & 0,13 & & $\mathrm{~ns}$ & $\mathrm{~ns}$ & $\mathrm{~ns}$ \\
\hline & \multicolumn{14}{|c|}{ Sumatorias } \\
\hline Saturados & 65,55 & 67,09 & 65,25 & 66,14 & 66,73 & 65,85 & & 67,83 & & 64,78 & & $\mathrm{~ns}$ & ns & ns \\
\hline Insaturados & 34,45 & 32,91 & 34,75 & 33,86 & 33,27 & 34,15 & & 32,17 & & 35,22 & & $\mathrm{~ns}$ & ns & ns \\
\hline Monoinsaturados & 30,38 & 28,90 & 30,26 & 29,63 & 29,13 & 30,01 & & 28,20 & & 30,78 & & $\mathrm{~ns}$ & ns & $\mathrm{ns}$ \\
\hline Poliinsaturados & 4,07 & 4,01 & 4,49 & 4,23 & 4,14 & 4,14 & & 3,97 & & 4,44 & & ns & $\mathrm{ns}$ & ns \\
\hline Omega $3(n-3)$ & 0,63 & 0,60 & 0,57 & 0,64 & 0,60 & 0,56 & $\mathrm{a}$ & 0,58 & $\mathrm{a}$ & 0,80 & $\mathrm{~b}$ & ns & 0,0209 & ns \\
\hline Omega 6 (n-6) & 1,67 & 1,71 & 1,85 & 1,79 & 1,66 & 1,64 & & 1,64 & & 1,92 & & ns & ns & $\mathrm{ns}$ \\
\hline $\mathrm{n} 6 / \mathrm{n} 3$ & 2,80 & 2,78 & 2,47 & 3,00 & 2,84 & 3,09 & $\mathrm{a}$ & 2,91 & $\mathrm{a}$ & 2,34 & $\mathrm{~b}$ & ns & 0,0055 & $\mathrm{~ns}$ \\
\hline $\mathrm{C} 10$ a C16 & 44,50 & 47,91 & 46,28 & 47,06 & 48,92 & 47,07 & $a b$ & 50,10 & $\mathrm{a}$ & 43,64 & $\mathrm{~b}$ & ns & 0,0456 & ns \\
\hline Trans C18:1 & 6,18 & 5,75 & 5,19 & 5,49 & 5,61 & 5,87 & & 5,42 & & 5,64 & & ns & ns & $\mathrm{ns}$ \\
\hline $\begin{array}{l}\text { Aterogénicos C12, } \\
14,16 \\
\text { Índice de }\end{array}$ & 39,82 & 43,22 & 41,79 & 42,41 & 44,26 & 42,16 & & 45,17 & & 39,56 & & ns & $\mathrm{ns}$ & ns \\
\hline aterogenicidad & 2,59 & 2,77 & 2,64 & 2,77 & 2,79 & 2,70 & & 3,09 & & 2,35 & & $\mathrm{~ns}$ & $\mathrm{~ns}$ & $\mathrm{~ns}$ \\
\hline
\end{tabular}

$\mathrm{F} * \mathrm{~T}$ : finca * tercio de lactancia. Valores con letras diferentes en la misma fila difieren significativamente entre tratamientos $(\mathrm{p}<0,05) /$ $\mathrm{F}^{*} \mathrm{~T}$ : farm * stage of lactation. Values with different letters in the same row differ significantly between treatments $(\mathrm{p}<0.05)$. 
consumido tuvo la mayor participación, seguido del ácido linolénico (Cuadro 5), lo que corresponde con altos valores de ALC-c9t11 para las fincas Esperanza y San Felipe (2,09 y 2,08, respectivamente). No obstante, la finca Pradera, que mostró el mayor porcentaje estimado de ácido linoleico consumido en el concentrado $(30,78 \%)$ y mayor consumo de linoleico respecto a las otras dos fincas, presentó el menor valor de ALC-c9t11 $(1,28 \%)$ y de ATV $(5,09 \%)$ de estas tres fincas, debido posiblemente a que en esta finca se suministra semilla de algodón entera (rica en ácido linoleico) como parte del concentrado. Igual situación ocurre en la finca Maracaibo del sistema DP, donde también se ofrece semilla de algodón entera, en esta finca se estimó que el ácido linoleico participó en $28,15 \%$ del total de grasa consumida, mayor al $18,43 \%$ estimado para la finca Vargas (Cuadro 6); sin embargo, el valor de ALC-c9t11 en la leche de Maracaibo fue menor que en Vargas $(1,98 \%$ vs 2,22). Esta observación está de acuerdo con lo encontrado por Rico et al. (2007), quienes reportaron una asociación negativa $(r=-0,7, p<0,05)$ entre la semilla de algodón y el contenido de ALC-c9t11, indicando una tendencia a la disminución del ALC-c9t11 al suplementar con semilla de algodón. Sin embargo, Aprianita et al. (2014), resaltan la bondad del aceite de semilla de algodón para aumentar ALC-c9t11 y ATV en leche.

En las fincas Pradera (LT) y Maracaibo (DP), en las cuales se suministró semilla de algodón entera, la proporción de ácido linoleico en leche fue mayor, con diferencias significativas en la finca Maracaibo del sistema DP. Lo anterior demuestra que el ácido linoleico presente en la semilla de algodón puede aumentar linoleico en leche, pero presenta dificultad para aumentar ALC-c9t11. Venturelli et al. (2015) reportaron que semillas enteras de oleaginosas sin procesamiento pueden producir una menor concentración de ALC-c9t11 y ATV en leche que las semillas procesadas o el aceite, debido a una menor disponibilidad de los ácidos grasos insaturados que hay en la semilla para que sean biohidrogenados en el rumen, dando como resultado un mayor contenido de ácidos grasos polinsaturados en leche.

En el sistema LTSSPi el porcentaje estimado de ácido linoleico del concentrado fue bajo entre 18,28\% y $23,25 \%$ del total de grasa consumida; el porcentaje total de oleico, linoleico y linolenico consumido fue más bajo que en los otros sistemas (Cuadro 5), por tanto, los valores de ALC-c9t11 en leche en este sistema fueron menores. Aunque en este sistema existió un consumo de leucaena entre 16 y $26 \%$ de la MS del forraje consumido diariamente, su aporte sobre aumento de ALC-c9t11 y ATV no marcó la diferencia en estos AG, dada la alta participación del concentrado. Asimismo, en la finca Hatico de este sistema, el ácido oleico presentó la mayor participación de los ácidos grasos precursores consumidos, por lo que esta finca marcó la diferencia en este sistema, con una mayor proporción de ácido oleico, monoinsaturados y menor índice de aterogenicidad en la leche. El alto consumo de oleico en la finca Hatico, aumentó la proporción de este ácido graso en leche, pero no aumentó los valores de ALC-c9t11, ni de ATV en leche de manera significativa, lo que está de acuerdo con lo reportado por Rego et al. (2009), quienes indicaron que ATV no es el principal producto de la isomerización de oleico en rumen; también está de acuerdo con el estudio de Stoffel et al. (2015), en el que al suplementar con aceite de girasol rico en ácido oleico, aumentó la proporción total de C18:1 en leche.

En el sistema DP, aunque la proporción de ácido linoleico consumido fue mayor en la finca Maracaibo (Cuadro 6), no se presentaron diferencias entre ALCc9t11 y ATV en la leche, debido posiblemente a la dificultad del ácido linoleico presente en la semilla de algodón para aumentar ALC-c9t11.

En el sistema DPSSPi que no usó suplementación y los AG presentes en la leche solo provenían del forraje, la edad del forraje y su efecto sobre la disponibilidad de la grasa a nivel de rumen, así como el consumo de leucaena, cobran importancia. La finca Salsipuedes cosechó el forraje a mayor edad que la finca Pradera (47 vs 31 días). Asimismo, en la finca Salsipuedes se presentó un bajo consumo de leucaena con respecto a Pradera (3\% vs 16\%), que representó 0,24 vs 1,6 kg de MS/animal/día, respectivamente; no obstante, el contenido de grasa y de ácido linolénico fue mayor que el de Pradera, razón por la cual el consumo total estimado de ácido linolénico y linoleico fue similar (Cuadro 6). Sin embargo, la finca Salsipuedes presentó una menor proporción ALCc9t11 en leche, aunque no significativa, la cual pudo deberse a una pobre disponibilidad a nivel ruminal de los AG poliinsaturados ingeridos, ocasionada por la edad de los forrajes, lo que también se refleja en menor ATV y menor proporción de trans C18:1 producto de la biohidrogenación; no obstante, el total de AG saturados, insaturados, omega 3, omega 
6, aterogénicos e índice de aterogenicidad no se vieron afectados. Ferlay et al. (2006) reportaron una disminución $(\mathrm{p}<0,01)$ en ALC-c9t11 de 17,2 a 8 $\mathrm{mg} / \mathrm{g}$ de grasa, cuando se cambió de 21 a 42 días de rebrote en una pastura nativa conformada por $50 \%$ de Festuca rubra. En la Sabana de Bogotá se encontró que el contenido de ALC-c9t11 en la leche de vacas en pastoreo de kikuyo disminuyó $(\mathrm{p}<0,01)$ de 22,4 a $14,4 \mathrm{mg} / \mathrm{g}$ de grasa al aumentar la edad de rebrote de cincuenta a setenta días (Aguilar et al., 2009).

Cuando se alimenta con altas dosis de AG insaturados se ha observado una disminución en la grasa de la leche, relacionada con un cambio en los procesos microbianos que implica una alteración en las vías y en la integridad de la biohidrogenación, lo cual resulta en un aumento en la formación de C18:1 trans10 y de intermediarios relacionados (Griinari et al., 1998), estos están altamente correlacionados con aumentos en el contenido en la grasa de la leche de C18:1 trans y de isómeros de CLA (Loor et al., 2005; Kadegowda et al., 2008; Shingfield et al., 2008). Asimismo, con este tipo de suplemetación pueden haber cambios en la expresión de genes lipogénicos en glándula mamaria, que provocan una disminución en la expresión de genes envueltos en la síntesis de la grasa (Angulo et al., 2012b). En este estudio, otros ALC como el C18:2 c10t12, C18:2 t10c12 y mezcla de ALC, no fueron detectados o su contenido fue más bajo que el nivel de detección y solo se registraron en pocas muestras, no ofreciendo información consistente para ser analizada.

Existe evidencia de que los AG saturados de la dieta aumentan las concentraciones de colesterol asociado a las lipoproteínas de baja densidad (LDL) (Givens, 2010). Los AG saturados laúrico, mirístico y palmítico pueden provocar aterosclerosis en seres humanos; teniendo el ácido mirístico, un efecto cuatro veces más fuerte que el palmítico sobre los niveles plasmáticos del colesterol (Ulbricht y Southgate, 1991), razón por la que se busca disminuir estos AG en la leche y aumentar los AG insaturados, buscando un índice de aterogenicidad bajo. La proporción de AG insaturados en la leche de las fincas en estudio fue similar a la registrada en otros estudios realizados con dietas en base a forrajes frescos (Rico et al., 2007; Vargas et al., 2013), pero superior a la registrada en estudios con forrajes conservados (Ferlay et al., 2010; Ferlay et al., 2013), debido al mayor suministro de ácido linolénico y linoleico por parte de los forrajes frescos y de los suplementos utilizados. Es de destacar la finca Hatico con $41,53 \%$ de AG insaturados y solo $58,46 \%$ de AG saturado, esta finca presentó un mayor suministro de grasa, con una mayor proporción de oleico en el suplemento (Cuadro 5), que se refleja en una mayor proporción de monoinsaturados en la leche, conllevando a una mayor proporción de insaturados en la leche y menor proporción de aterogénicos e índice de aterogenicidad.

Los AG n-3 y n-6 son esenciales en la dieta, ya que son necesarios para las funciones fisiológicas normales ligadas a la integridad de membrana y a señales reguladoras de las células; el equilibrio de AG n-6 y n-3 en la dieta es un factor crítico que influye en la salud humana (Wijendran y Hayes, 2004). Los efectos beneficiosos de los AG poliinsaturadas n-3 están bien documentados, y el aumento en su consumo reduce el riesgo de enfermedad cardiovascular, mejora el desarrollo fetal y puede proteger contra la demencia (Givens y Gibbs, 2008). Se ha recomendado aumentar la ingesta de AG n-3 y disminuir la ingesta de AG n-6, basado en la preocupación de que el alto consumo de AG n-6 pueden interferir con el metabolismo de los AG n-3, y se puede aumentar el riesgo de enfermedades inflamatorias, trastornos del sistema inmune y aumentan la susceptibilidad de los lípidos del tejido a una modificación oxidativa (Calder, 2006; ADS, 2007); se ha recomendado mantener una relación n-6/n-3, menor de 5:1 (WHO, 2003). En salud humana resulta más conveniente utilizar la relación omega $6 / 3$, que la concentración individual absoluta de ellos en la ración (Gagliostro, 2011). En este estudio, la leche de las fincas del sistema DPSSPi, donde su alimentación fue solo a base de forraje y no recibían suplemento, presentaron una baja relación n6/n3 (2,68 y 2,88), ocasionada por el alto consumo de linolénico (n3) proveniente del forraje; en las fincas de los sistemas LT y DP que recibían suplementación rica en ácido linoleico (n6), esta relación estuvo en torno a cinco y solo en la leche del sistema LTSSPi esta relación fue superior a cinco, situación susceptible de mejorar con una suplementación de ácidos grasos que busque este objetivo.

Los ácidos láurico, mirístico y palmítico cuando son consumidos en exceso elevan el colesterol plasmático total y el colesterol asociado a las LDL (Schrezenmeir y Jagla, 2000), a pesar de que el riesgo de aterosclerosis por el consumo de grasas lácteas no está definido (Chowdhury et al., 2014), los productos 
lácteos con índice aterogénicos inferiores tienen menos probabilidad que sean negativos para la salud humana (Ulbricht y Southgate, 1991; Allred et al., 2006). El índice aterogénico de las leches en este estudio estuvo entre 2,12 a 2,89 y en la finca Hatico en 1,69. VargasBello-Pérez et al. (2015) reportaron un valor de 2,50 para leche de vacas alimentadas con dietas TMR a base de forrajes conservados y de 1,55 para leche de vacas alimentadas con TMR, pero suplementadas con $500 \mathrm{~g} / \mathrm{animal} /$ día de aceite de soja.

\section{Concentración de ácidos grasos en la leche y número de partos}

El efecto del número de parto sobre los AG insaturados de la leche, se presentó con muy poca frecuencia, solo para AG oleico en el sistema LTSSPi (Cuadro 10) y para AG linolénico en el sistema DP (Cuadro 12). De La Fuente et al. (2009) sugieren que la influencia del número de partos sobre el contenido de ácidos grasos en la leche es mínimo. Asimismo, Kelsey et al. (2003) concluyeron que los factores fisiológicos como número de partos tienen poca influencia sobre el contenido de ALC en la leche cuando se compara frente a los factores dietarios.

\section{Concentración de ácidos grasos en la leche y tercio de lactancia}

Investigadores han sugerido que al aumentar los días de lactancia se presenta variación en la composición de la grasa láctea, evidenciándose en los primeros días una mayor concentración de ácidos grasos de cadena larga provenientes de la movilización de las reservas corporales $\mathrm{y}$, posteriormente, el incremento en los ácidos que se obtienen por síntesis de novo (Kelsey et al 2003; Bargo et al., 2006). Sin embargo, Mapekula et al. (2011) observaron concentraciones más altas de ácidos grasos saturados en la etapa temprana de la lactancia en comparación con mediados y finales de esta.

En dos investigaciones se encontró un aumento en la concentración de ALC en la medida que se incrementaban los días en lactancia (Kay et al., 2005; Stoop et al., 2009). En otro estudio se informó que el perfil de ácidos grasos, con excepción al ALC-c9t11, no fue modificado por el tercio de lactancia y este ácido graso presentó mayores concentraciones en animales de segundo tercio al compararlos con los de primer tercio (Vargas et al., 2013). Los resultados de este estudio, están de acuerdo con los mencionados anteriormente, en el sentido que se presentó diferencia por tercio de lactancia para la proporción de ALC-c9t11 en los sistemas LT (Cuadro 8) y DP (Cuadro 12), presentando un valor mayor en el segundo y tercer tercio con respecto al primero. Para los demás ácidos grasos insaturados evaluados no se presentó una variación consistente por tercio de lactancia que afectara el índice de aterogenicidad, contrario a lo observado por Nantapo et al. (2014), quienes reportaron una menor proporción de AG insaturados en lactancia tardía, lo que llevó a un alto índice de aterogenicidad.

\section{AGRADECIMIENTOS}

Los autores de este trabajo agradecen el financiamiento realizado por Colciencias (Proyecto Colciencias No. PRE00503029606), Universidad de Caldas, Universidad de Antioquia (UdeA) y la Universidad de Sucre. A los ganaderos Carlos Alberto Gómez Buendía (Finca Asturias), Milciades Salas (Finca Pradera), Andrés Jaramillo Bernal (Finca Maracaibo), Roberto Gutiérrez (Fincas Vargas y Japón), Claudia Patricia Arcila (Finca Esperanza), Juan José Molina y Flia (Finca Hatico), Salvador Raad (Finca Salsipuedes), Abraham Ovalle (finca Campo Alegre), a los administradores Juan Fernando Suárez (Finca Lucerna), José Fernando Cardona (Finca San Felipe), Alirio Nieto (Finca La Pradera) y a sus trabajadores, quienes muy gentilmente, apoyaron el desarrollo de este trabajo. Asimismo, resaltan y agradecen el apoyo técnico de los profesores Mario Cerón, Oscar Vergara, Wilson Barragán, y la colaboración y el acompañamiento de la Fundación Centro para la Investigación en Sistemas Sostenibles de Producción Agropecuaria (CIPAV) en el desarrollo del proyecto.

\section{LITERATURA CITADA}

ADS (Ammerican Dietetic Assocition). 2007. Position of the American Dietetic Association and Dietitians of Canada: dietary fatty acids. J. Am. Diet. Assoc. 107: 1599-1611.

Aguilar, O.X., B.M. Moreno, M.L. Pabón, y J.E. Carulla. 2009. Efecto del consumo de kikuyo (Pennisetum 
clandestinum) o raigrás (Lolium hibridum) sobre la concentración de ácido linoléico conjugado y el perfil de ácidos grasos de la grasa láctea. Livest. Res. Rural Dev. 21(4). http://www.lrrd.org/lrrd21/4/agui21049. htm (consultado 29 nov. 2015).

Allred, S.L., T.R. Dhiman, C.P. Brennand, R.C. Khanal, D.J. McMahon, and N.D. Luchini. 2006. Milk and cheese from cows fed calcium salts of palm and fish oil alone or in combination with soybean products. J. Dairy Sci. 89:234-248.

Angulo, J., B. Hiller, M. Olivera, L. Mahecha, D. Dannenberger, G. Nuernberg, B. Losand, and K. Nuernberg. 2012a. Dietary fatty acid intervention of lactating cows simultaneously affects lipid profiles of meat and milk. J. Sci. Food Agric. 92:2968-2974.

Angulo, J., L. Mahecha, K. Nuernberg, G. Nuernberg, D. Dannenberger, M. Olivera, M. Boutinaud, C. Leroux, E. Albrecht, and L. Bernard. 2012b. Effects of polyunsaturated fatty acids from plant oils and algae on milk fat yield and composition are associated with mammary lipogenic and SREBF1 gene expression. Animal 6:1961-1972.

AOAC (Association of Official Analytical Chemist). 1999. Official methods of analysis. $16^{\text {th }}$ ed. AOAC International, Gaithersburg, MD, USA.

Aprianita, A., O.N. Donkor, P.J. Moate, S.R.O. Williams, M.J. Auldist, J.S. Greenwood, M.C. Hannah, W.J. Wales, and T. Vasiljevic. 2014. Effects of dietary cottonseed oil and tannin supplements on protein and fatty acid composition of bovine milk. J. Dairy Res. 81:183-192.

Bacab-Pérez, H.M., y F.J. Solorio-Sánchez. 2011. Oferta y consumo de forraje y producción de leche en ganado de doble propósito manejado en sistemas silvopastoriles en Tepalcatepec, Michoacán. Trop. Subtrop. Agroecosyst. 13:271-278.

Bargo, F., J. Delahoy, G. Schroeder, and L. Muller. 2006. Milk fatty acid composition of dairy cows grazing at two pasture allowances and supplemented with different levels and sources of concentrate. Anim. Feed Sci. Technol. 125:17-31.

Bichi, E., P.G. Toral, G. Hervás, P. Frutos, P. Gómez-Cortés, M. Juárez, and M.A. De la Fuente. 2012. Inhibition of $\Delta$ 9-desaturase activity with sterculic acid: effect on the endogenous synthesis of cis-9 18:1 and cis-9, trans-11 18:2 in dairy sheep. J. Dairy Sci. 95:5242-5252.

Boerman, J.P., and A.L. Lock. 2014. Effect of unsaturated fatty acids and triglycerides from soybeans on milk fat synthesis and biohydrogenation intermediates in dairy cattle. J. Dairy Sci. 97:7031-7042.

Calderón, A., F. García, y G. Martínez. 2006. Indicadores de calidad de leches crudas en diferentes regiones de Colombia. Rev. MVZ Córdoba 11:725-737.

Calder, P.C. 2006. Polyunsaturated fatty acids and inflammation. PLEFA 75:197-202.

Campabadall, C. 1999. Factores que afectan el contenido de sólidos de la leche. Nutr. Anim. Trop. 5(1):67-92.

Chilliard, Y., and A. Ferlay. 2004. Dietary lipids and forages interactions on cow and goat milk fatty acid composition and sensory properties. Reprod. Nutr. Dev. 44:467-92.

Chilliard, Y., F. Glasser, A. Ferlay, L. Bernard, J. Rouel, and M. Doreau. 2007. Diet, rumen biohydrogenation and nutritional quality of cow and goat milk fat. Eur. J. Lipid Sci. Technol. 109:828-855.

Chowdhury, R., S. Warnakula, S. Kunutsor, F. Crowe, H. Ward, L. Johnson, O. Franco, A. Butterworth, N. Forouhi, S. Thompson, K. Khaw, D. Mozaffarian, J. Danesh, and E. Angelantonio. 2014. Association of dietary, circulating, and supplement fatty acids with coronary risk: a systematic review and meta-analysis. Ann. Intern. Med. 160:398-406.

Christie, W. 1990. Fatty acids and lipids: structures, extraction and fractionation into classes. In: W.W. Christie, editor, Gas chromatography and lipids: a practical guide. The Oily Press. p. 5-23.

CONPES (Consejo Nacional de Política Económica y Social). 2010. Política nacional para mejorar la competitividad del sector lácteo colombiano. Documento 3675. CONPES, Bogotá D.C., COL.

Cozma, A., B. Martin, E. Tixier, M. Guiadeur, P. Pradel, S. Andrei, and A. Ferley. 2011. Effect of calf presence during milking on milk fatty acid profile in Prim'holstein and salers cow breeds. Bull. UASVM 68:118-120.

De La Fuente, L.F., E. Barbosa, J.A. Carriedo, C. Gonzalo, R. Arenas, J.M. Fresno, and F. San Primitivo. 2009. Factors influencing variation of fatty acid content in ovine milk. J. Dairy Sci. 92:3791-3799.

Dewhurst, R.J., K.J. Shingfield, M.R.F. Lee, and N.D. Scollan. 2006. Increasing the concentrations of beneficial polyunsaturated fatty acids in milk produced by dairy cows in high-forage systems. Anim. Feed Sci. Technol. 131:168-206.

Elgersma, A., S. Tamminga, and G. Ellen. 2006. Modifying milk composition through forage. Anim. Feed Sci. Tecnol. 131:207-225. 
Ferlay, A., M. Doreau, C. Martin, and Y. Chilliard. 2013. Effects of incremental amounts of extruded linseed on the milk fatty acid composition of dairy cows receiving hay or corn silage. J. Dairy Sci. 96:6577-6595.

Ferlay, A., B. Martin, S. Lerch, M. Gobert, P. Pradel, and Y. Chilliard. 2010. Effects of supplementation of maize silage diets with extruded linseed, vitamin E and plant extracts rich in polyphenols, and morning v. evening milking on milk fatty acid profiles in holstein and montbéliarde cows. Anim. 4:627-640.

Ferlay, A., B. Martin, P. Pradel, J.B. Coulon, and Y. Chilliard. 2006. Influence of grass-based diets on milk fatty acid composition and milk lipolytic system in tarentaise and montbeliarde cow breeds. J. Dairy Sci. 89:4026-4041.

Fröberg, S., A. Aspegren-Güldorff, I. Olsson, B. Marin, C. Berg, C. Hernández, C.S. Galina, L. Lidfors, and K. Svennersten-Sjaunja. 2007. Effect of restricted suckling on milk yield, milk composition and udder health in cows and behaviour and weight gain in calves, in dual-purpose cattle in the tropics. Trop. Anim. Health Prod. 39:71-81.

Gagliostro, G.A. 2011. Obtención de lácteos funcionales naturales. En: C.A. Cangiano, y M.A. Brizuela, editores, Producción animal en pastoreo. $2^{\text {da }}$ ed. INTA, Balcarce, ARG. p. 425-445.

Givens, D.I. 2010. Milk and meat in our diet: good or bad for health? Anim. 4:1941-1952.

Givens, D.I., and R.A. Gibbs. 2008. Current intakes of EPA and DHA in European populations and the potential of animal-derived foods to increase them. Proc. Nutr. Soc. 67:273-280.

Griinari, J.M., and D.E. Bauman. 1999. Biosynthesis of conjugated linoleic acid and its incorporation into meat and milk in ruminants. In: M.P. Yurawecz et al., editors, Advances in CLA Research, vol. 1. AOCS Press, Champaign, IL, USA. p. 180-200.

Griinari, J.M., D.A. Dwyer, M.A. McGuire, D.E. Bauman, D.L. Palmquist, and K.V Nurmela. 1998. Transoctadecenoic acids and milk fat depression in lactating dairy cows. J. Dairy Sci. 81:1251-1261.

Harris, W.S. 2008. Linoleic acid and coronary heart disease. PLEFA 79:169-171.

Hervás, G., P. Luna, A.R. Mantecon, N. Castañares, P. Frutos, M.A. De la Fuente, and M. Juárez. 2006. Effect of sunflower oil on sheep milk production and composition, and in vitro rumen fermentation. In: European Federation for the Science and Technology of Lipids, editor, $4^{\text {th }}$ Euro Fed Lipid Congress: Fats, oils and lipids for a healthier future. German Chemical Society (GDCh), Madrid, ESP. p. 18.

Kadegowda, A.K.G., L.S. Piperova, and R.A. Erdman. 2008. Principal component and multivariate analysis of milk long-chain fatty acid composition during diet-induced milk fat depression. J. Dairy Sci. 91:749-759.

Khanal, R.C., and K.C. Olson. 2004. Factors affecting conjugated linoleic acid (CLA) content in milk, meat, and egg: A review. Pakistan J. Nutr. 3:2-98.

Kay, J.K., W.J. Weber, C.E. Moore, D.E. Bauman, L.B. Hansen, H. Chester-Jones, B.A. Crooker, and L.H. Baumgard. 2005. Effects of week of lactation and genetic selection for milk yield on milk fatty acid composition in holstein cows. J. Dairy Sci. 88:3886-3893.

Kelsey, J.A., B.A. Corl, R.J. Collier, and D.E. Bauman. 2003. The effect of breed, parity, and stage of lactation on conjugated linoleic acid (CLA) in milk fat from dairy cows. J. Dairy Sci. 86:2588-2597.

Kepler, C.R., and S.B. Tove. 1967. Biohydrogenation of unsaturated fatty acids: III. Purification and properties of a linoleate $\Delta 12$-Cis, $\Delta 11$-trans-isomerase from Butyrivibrio fibrisolvens. J. Biol. Chem. 242:5686-5692.

Khiaosa-Ard, R., S.F. Bryner, M.R.L. Scheeder, H.R. Wettstein, F. Leiber, M. Kreuzer, and C.R. Soliva. 2009. Evidence for the inhibition of the terminal step of ruminal alpha-linolenic acid biohydrogenation by condensed tannins. J. Dairy Sci. 92:177-188.

Lawless, F., C. Stanton, P. L'Escop, R. Devery, P. Dillon, and J.J. Murphy. 1999. Influence of breed on bovine milk cis-9, trans-11-conjugated linoleic acid content. Livest. Prod. Sci. 62:43-49.

Loor, J.J., A. Ferlay, A. Ollier, M. Doreau, and Y. Chilliard. 2005. Relationship among trans and conjugated fatty acids and bovine milk fat yield due to dietary concentrate and linseed oil. J. Dairy Sci. 88:726-740.

Macoon, B., L.E. Sollenberger, J.E. Moore, C.R. Staples, J.H. Fike, and K.M. Portier. 2003. Comparison of three techniques for estimating the forage intake of lactating dairy cows on pasture. J. Anim. Sci. 81:2357-2366.

Mahecha, L., C.V Durán, M. Rosales, C.H. Molina, y E. Molina. 2000. Consumo de pasto Estrella Africana (Cynodon plectostachyus) y leucaena (Leucaena leucocephala) en un sistema silvopastoril. Pasturas Trop. 22:26-30.

Mapekula, M., M. Chimonyo, C. Mapiye, and K. Dzama. 2011. Fatty acid, amino acid and mineral composition 
of milk from nguni and local crossbred cows in South Africa. J. Food Compos. Anal. 24:529-536.

Mele, M., A. Buccioni, F. Petacchi, A. Serra, S. Banni, M. Antongiovanni, and P. Secchiari. 2006. Effect of forage/ concentrate ratio and soybean oil supplementation on milk yield, and composition from Sarda ewes. Anim. Res. 55(4):273-285.

Murgueitio, E. 1999. Environmental and social adjustment of the cattle farming sector in Colombia. World Anim. Rev. 93:2-15.

Murgueitio, E., y M. Ibrahim. 2008. Ganadería y medio ambiente en América Latina. En: E. Murgueitio et al., editores, Ganadería del futuro: investigación para el desarrollo. $2^{\text {da }}$ ed. Fundación CIPAV, Cali, COL. p. 19-40.

Nantapo, C.T.W., V. Muchenje, and A. Hugo. 2014. Atherogenicity index and health-related fatty acids in different stages of lactation from friesian, jersey and friesian $\mathrm{x}$ jersey cross cow milk under a pasture-based dairy system. Food Chem. 146:127-133.

Ojeda, A., A. Lugo, y P. Petrocínio. 2001. Evaluación de la concentración de grasa en leche de vacas de doble propósito con amamantamiento restringido. Rev. Unellez Cienc. Tecnol. Especial:1-5.

Pagiola, S., P. Agostini, J. Gobbi, C. de Haan, M. Ibrahim, E. Murgueitio, E. Ramírez, M. Rosales, and J.P. Ruíz. 2005. Paying for biodiversity conservation services: experience in Colombia, Costa Rica, and Nicaragua. Mt. Res. Dev. 25:206-211.

Pagiola, S., E. Ramirez, J. Gobbi, C. De Haan, M. Ibrahim, E. Murgueitio, and J.P. Ruiz. 2007. Paying for the environmental services of silvopastoral practices in Nicaragua. Ecol. Econ. 64:374-385.

Palmquist, D.L. 2007. Biohydrogenation then and now. Eur. J. Lipid Sci. Technol. 109:737-739.

Prendiville, R., K.M. Pierce, L. Delaby, and F. Buckley. 2011. Animal performance and production efficiencies of holstein-friesian, jersey and jersey $\times$ holstein-friesian cows throughout lactation. Livest. Sci. 138:25-33.

Rego, O.A., S.P. Alves, L.M.S. Antunes, H.J.D. Rosa, C.F.M. Alfaia, J.A. M. Prates, A.R.J. Cabrita, A.J.M. Fonseca, and R.J.B. Bessa. 2009. Rumen biohydrogenationderived fatty acids in milk fat from grazing dairy cows supplemented with rapeseed, sunflower, or linseed oils. J. Dairy Sci. 92:4530-4540.

Restrepo, J. 2013. Estimación del consumo de forraje de vacas lecheras pastoreando en sistemas silvopastoriles intensivos (SSPi) en el bosque seco tropical Colombiano. Rev. Colomb. Cienc. Pecu. 26:441.
Rico, J.E., B. Moreno, M.L. Pabón, y J. Carulla. 2007. Composición de la grasa láctea en la sabana de Bogotá con énfasis en ácido ruménico - CLA cis -9, trans -11. Rev. Col. Cienc. Pecu. 20:30-39.

Saliba, L., R. Gervais, Y. Lebeuf, and P.Y. Chouinard. 2014. Effect of feeding linseed oil in diets differing in forage to concentrate ratio: 1 . Production performance and milk fat content of biohydrogenation intermediates of $\alpha$-linolenic acid. J. Dairy Res. 81:82-90.

SAS. 2001.Version 8.2. User's guide. SAS Institute Inc., Cary, NC, USA.

Schrezenmeir, J., y A. Jagla. 2000. Milk and diabetes. J. Am. Coll. Nutr. 19:176-190.

Schroeder, G.F., G.A. Gagliostro, F. Bargo, J.E. Delahoy, and L.D. Muller. 2004. Effects of fat supplementation on milk production and composition by dairy cows on pasture: a review. Livest. Prod. Sci. 86:1-18.

Shingfield, K.J., M. Bonnet, and N.D. Scollan. 2013. Recent developments in altering the fatty acid composition of ruminant-derived foods. Anim. 7:132-162.

Shingfield, K.J., Y. Chilliard, V. Toivonen, P. Kairenius, and D.I. Givens. 2008. Trans fatty acids and bioactive lipids in ruminant milk. Adv. Exp. Med. Biol. 606:3-65.

Shingfield, K.J., C.K. Reynolds, G. Hervás, J.M. Griinari, A.S. Grandison, and D.E. Beever. 2006. Examination of the persistency of milk fatty acid composition responses to fish oil and sunflower oil in the diet of dairy cows. J. Dairy Sci. 89:714-732.

Stoffel, C.M., P.M. Crump, and L.E. Armentano. 2015. Effect of dietary fatty acid supplements, varying in fatty acid composition, on milk fat secretion in dairy cattle fed diets supplemented to less than $3 \%$ total fatty acids. J. Dairy Sci. 98:431-442.

Stoop, W.M., H. Bovenhuis, J.M.L. Heck, and J.A.M. Van Arendonk. 2009. Effect of lactation stage and energy status on milk fat composition of holstein-friesian cows. J. Dairy Sci. 92:1469-1478.

Tequin-Ocampo, E. 2014. Estudio de la influencia de la suplementación lipídica en la dieta de bovinos sobre los ácidos grasos funcionales de la leche, y la producción de metano y ácidos grasos volátiles del fluido ruminal por cromatografía de gases. Tesis M.Sc., Universidad de Caldas, COL.

Ulbricht, T.L., and D.A. Southgate. 1991. Coronary heart disease: seven dietary factors. Lancet 338:985-992.

Van Soest, P.J., J.B. Robertson, and B.A. Lewis. 1991. Methods for dietary fiber, neutral detergent fiber, and nonstarch polysaccharides in relation to animal nutrition. J. Dairy Sci. 74:3583-3597. 
Vargas-Bello-Pérez, E., K. Fehrmann-Cartes, G. ÍñiguezGonzález, P. Toro-Mujica, and P.C. Garnsworthy. 2015. Chemical composition, fatty acid composition, and sensory characteristics of chanco cheese from dairy cows supplemented with soybean and hydrogenated vegetable oils. J. Dairy Sci. 98:111-117.

Vargas M, J., J. Mojica, M. Pabón, y J. Carulla. 2013. Oferta de pasto kikuyo (Pennisetum clandestinum), tercio de lactancia y perfil de ácidos grasos lácteos. Rev. MVZ Córdoba 18:3681-3688.

Venturelli, B.C., J.E. de Freitas Jr., C.S. Takiya, A.P.C. de Araújo, M.C.B. Santos, G.D. Calomeni, R. Gardinal, T.H.A. Vendramini, and F.P. Rennó. 2015. Total tract nutrient digestion and milk fatty acid profile of dairy cows fed diets containing different levels of whole raw soya beans. J. Anim. Physiol. Anim. Nutr. 99:1149-60.

Wallace, R. J., N. McKain, K.J. Shingfield, and E. Devillard. 2007. Isomers of conjugated linoleic acids are synthesized via different mechanisms in ruminal digesta and bacteria. J. Lipid Res. 48:2247-2254. doi:10.1194/jlr.M700271-JLR200
Weiss, M.F., F.A. Martz, and C.L. Lorenzen. 2004a. Conjugated linoleic acid: historical context and implications. Prof. Anim. Sci. 20:118-126.

Weiss, M.F., F.A. Martz, and C.L. Lorenzen. 2004b. Conjugated linoleic acid: implicated mechanisms related to cancer, atherosclerosis, and obesity. Prof. Anim. Sci. 20:127-135.

White, S.L., J.A. Bertrand, M.R. Wade, S.P. Washburn, J.T. Green, and T.C. Jenkins. 2001. Comparison of fatty acid content of milk from jersey and holstein cows consuming pasture or a total mixed ration. J. Dairy Sci. 84:2295-2301.

WHO (World Health Organization). 2003. Diet, nutrition and the prevention of chronic diseases. Report of a joint $\mathrm{WHO} / \mathrm{FAO}$ expert consultation, series 916 . WHO Technical Report, Geneva, SWZ.

Wijendran, V., and K.C. Hayes. 2004. Dietary n-6 and n-3 fatty acid balance and cardiovascular health. Annu. Rev. Nutr. 24:597-615. 